\title{
Estimation and LQG control over unreliable network with acknowledgment randomly lost
}

\author{
Hong Lin, Hongye Su${ }^{\dagger}$, Senior Member, IEEE, Peng Shi, Fellow, IEEE, Renquan Lu, and Zheng-Guang Wu
}

\begin{abstract}
In this paper, we study the state estimation and optimal control (i.e., linear quadratic Gaussian (LQG) control) problems for networked control systems in which control inputs, observations, and packet acknowledgments are randomly lost. The packet acknowledgment is a signal that is sent from the actuator to inform the estimator whether the actuator has successfully received the control packets or not. For such systems, we obtain the optimal estimator, which is consisted of exponentially increasing terms. For the solvability of the optimal LQG problem, we come to a conclusion that even when the optimal LQG control exists, it is impossible and unnecessary to be obtained as its calculation is not only technically difficult but also computationally prohibitive. This issue motivates us to design a sub-optimal LQG controller for the underlying systems. We first develop a sub-optimal estimator by using the estimator gain in each term of the optimal estimator. Then we derive the suboptimal LQG controller and establish the conditions for stability of the closed-loop systems. Examples are given to illustrate the effectiveness and advantages of the proposed design scheme.
\end{abstract}

Index Terms-networked control systems, optimal estimation and control, LQG, packet loss, Quasi-TCP-like system

\section{INTRODUCTION}

Recently, increasing attention has been paid on the systems with their components (e.g., sensors, controllers, and actuators) connected via network, namely networked control systems (NCSs)[1]. The insertion of network brings numerous benifits, such as reduced system wiring, lower cost in maintenance, increased system agility, ease of information sharing, etc. However, it also causes some network-induced constraints, e.g., channel congestion, transmission delay, signal degradation, which may result in packet losses in data transmission $[2,3]$. To deal with this challenging issue, a number of techniques have been developed, such as the $H_{\infty}$ filter [48], the robust control [9-12], the predictive control [13, 14], and the fuzzy model-based approach $[15,16]$.

For NCSs with packet losses, two transport protocols are commonly deployed, i.e., the user datagram protocol (UDP)

Hong Lin, Hongye $\mathrm{Su}$, and Zheng-Guang $\mathrm{Wu}$ are with Institute of Cyber-Systems and Control, Zhejiang University, Hangzhou, Zhejiang, 310027, China. (e-mail: linhongzju@163.com, hysu@iipc.zju.edu.cn, nashwzhg@126.com) ${ }^{\dagger}$ : Corresponding author.

P. Shi is with the School of Electrical and Electronic Engineering, University of Adelaide, Adelaide, SA 5005, Australia. (email: peng.shi@adelaide.edu.au)

Renquan Lu is with the School of Automation, Guangdong University of Technology, and Guangdong Key Laboratory of IoT Information Processing, Guangzhou 510006, China. (e-mail:rqlu@hdu.edu.cn)

The work is supported in part by the National Natural Science Foundation of China $(61320106009,61134007,61573112,61374053)$, the 111 Project (B07031), Zhejiang Provincial Natural Science Foundation of China (R1100716), China National Funds for Distinguished Young Scientists (61425009), and Australian Research Council (DP140102180, LP140100471, LE150100079). and transmission control protocol (TCP). NCSs with different protocol lead to distinct results in estimation and control [17]. The key difference between these two protocols lies in the existence of acknowledgment mechanism. In NCS with TCP protocol, there are acknowledgments (ACKs) sent from the actuator to inform the estimator whether the actuator has successfully received the control packets or not. Such system is usually called as the TCP-like system. The NCS with UDP protocol, that is, in which there is no ACK available for the estimator, is called as the UDP-like system. For the UDP-like system, the implementation of transport protocol is simplified and the additional energy consumption for the ACK signal transmission is avoided. However, the lack of ACKs not only makes difficult the analysis of the UDP-like system, but also degrades the performances of estimation and control [18, 19]. For the TCP-like system, the ACK mechanism facilitates theoretical analysis, but it is reported that for NCSs over unreliable network, subject to network jitter and transmission delay, it is impossible to send the ACK in time (without delay and loss) to implement the TCP scheme [20-24]. Therefore, the NCS with ACK randomly lost turns out to be a reasonable and practical model for many applications, and such system is called as the Quasi-TCP-like system [23, 24]. In this paper, we are concerned with the optimal and sub-optimal solutions to the estimation and linear quadratic Gaussian (LQG) control problems for the Quasi-TCP-like systems.

For the TCP-like system, its optimal estimator has been early known as the time-varying Kalman filter, and its stability depends on a critical value for the observation packet loss rate [25]. The critical value together with its upper and lower bounds has been further studied in [26, 27]. For the LQG problem, it has been pointed out in $[17,28]$ that "the separation principle holds, and the optimal LQG controller is a linear function of the estimated state." Thereafter, the LQG problem for the TCP-like systems has been extensively studied for various cases [29-31]. While, these results generally do not hold for the Quasi-TCP- or UDP-like systems.

For the UDP-like system, the optimal estimator and its stability were studied in our recent work [18] for a special case: the UDP-like system without observation packet loss. The structure of the optimal estimator is complex and its computation is time-consuming. Possibly due to the complexity of the optimal estimator, to our best knowledge, the optimal LQG problem is rarely studied. Instead of the optimal estimator, the linear minimum mean square error (LMMSE) estimator was used in studying the LQG problem (the LMMSE-estimatorbased LQG problem). While it is still difficult to solve this problem except for some special cases [17]. Another sub- 
optimal linear LQG controllers were designed in [19, 21, 22]. However, in $[21,22]$ the finite horizon LQG problem is not studied, and the estimates used are the time-update prediction not the measurement-update estimation, which degrades the estimation and control performances. The controller propsed in [19] can stabilize the closed-loop system, only when the system is scalar and there is no observation packet loss.

For the Quasi-TCP-like system, an efficient sub-optimal estimator was developed in [32] but the optimal one is little studied. Thus, the LMMSE estimator was again used to study the LQG problem in [30], and the authors came to a conclusion that "in general, the separation principle does not hold, and the computation of the LQG controller requires solving a nonlinear optimization problem, and the resulting controller is a nonlinear function in the estimated state. If the observation equation is noise free and the matrix $C$ is square and invertible, then the optimal control is a linear function of the estimates." The main reason is that when the system state is estimated by the measurement-update estimator, like the LMMSE estimator, the estimation error covariance (ECC) will be a nonlinear function of the control $u_{k}$, making it difficult to solve the LQG problem. Although the time-update predictor not the measurement-update estimator was used in [24] to avoid such difficulty, the resulting structure of the ECC is still complicated. Thus, the author had to introduce some approaximations in the derivation to obtain a sub-optimal LQG controller.

As far as we know, the optimal estimator for the QuasiTCP-like system has not yet been obtained. Therefore, what is the structure of it? Whether does there exist a solution to the optimal LQG problem, and is the solution solvable? We also wonder that whether or not there is a sub-optimal measurement-update estimator, based on which the LQG problem can be solved and the resulting LQG controller can be presented in the traditional and familar way like the classic LQG controller. These questions, to our best knowledge, remain unsolved. Hence, motivated by these issues, we study the optimal as well as sub-optimal solutions to the estimation and LQG problems for the Quasi-TCP-like system. Our main results and contributions are summarized as follows:

- Optimal estimator: We derive the optimal estimator for the Quasi-TCP-like system, and show that its ECC can be decoupled into two parts: one is the EEC for the TCPlike system and the other is a summation consisting of exponentially increasing terms.

- Solvability of the optimal LQG problem: Up to now, it is little known about the solution to the optimal LQG problem. We make a conclusion on its solvability that in general, even when the optimal LQG control exists, it is impossible and unnecessary to obtain the optimal solutions for both the estimation and LQG control, as their calculations are not only technically difficult but also computationally prohibitive. We show that the difficulties in solving the optimal LQG problem differ from the well known ones to obtain the LMMSE-estimator-based LQG control.

- Sub-optimal LQG controller: Although the optimal estimator cannot be used in practice, in its complex structure

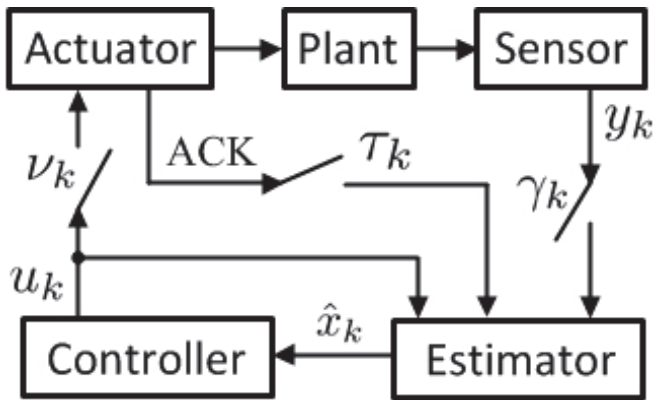

Fig. 1. The Quasi-TCP-like systems.

we find a component and then use it to design the desired sub-optimal estimator. Based on it, the LQG controller is obtained, which can be presented in the form similar to the classic LQG controller. Finally, conditions for the stability of the closed-loop systems are given.

The rest of the paper is organized as follows: In Section II, the system setup and problems are formulated. In Section III, the optimal estimator and the LQG problems are studied. Suboptimal estimator and LQG controller are developed in Section IV. In Section V, numerical examples are given to illustrate the effectiveness of the proposed design methods. The conclusions are presented in Section VI.

Notations:

- $\mathcal{N}_{x}(\mu, P)$ denotes the Gaussian pdf of the random variable $x$ with mean $\mu$ and covariance $P$.

- $x \sim \mathcal{N}_{x}(\mu, P)$ means that the pdf of the random variable $x$ is $\mathcal{N}_{x}(\mu, P)$.

- $\mathbb{P}(\cdot)$ denotes probability measure.

- $p(\cdot)$ and $p(\cdot \cdot \cdot)$ denote the pdf and the conditional pdf, respectively.

- $\mathbb{E}[\cdot]$ denotes probability expectation.

- $(\cdot)^{\prime}$ denotes the transpose of a matrix or a vector.

- $\operatorname{tr}(\cdot)$ denotes the trace of matrix.

- Let $M$ be a matrix. $(\cdot)_{M}^{2}$ denotes the quadratic form of $(\cdot) M(\cdot)^{\prime} .(\cdot)_{I}^{2}$ with the identity matrix $I$ means $(\cdot)(\cdot)^{\prime}$.

- $\lambda(M), \lambda_{M}: \lambda(M)$ denotes the spectral radius of $M$, and $\lambda_{M} \triangleq \lambda(M)$.

\section{SYSTEM SETUP AND PROBLEM FORMULATION}

\section{A. System setup}

Consider the following discrete-time Quasi-TCP-like linear system:

$$
\begin{aligned}
x_{k+1} & =A x_{k}+\nu_{k} B u_{k}+\omega_{k} \\
y_{k} & = \begin{cases}C x_{k}+v_{k}, & \text { for } \gamma_{k}=1 \\
\phi, & \text { for } \gamma_{k}=0\end{cases}
\end{aligned}
$$

where $x_{k} \in \mathbb{R}^{n}, u_{k} \in \mathbb{R}^{q}$, and $y_{k} \in \mathbb{R}^{p}$ are system state, control input, and observation, respectively. $\phi$ denotes empty set. $\omega_{k}$ and $v_{k}$ are zero mean Gaussian noises with covariances $Q \geq 0$ and $R>0$, respectively. $\nu_{k}, \gamma_{k}$, and $\tau_{k}$ (see, Fig. 1) are i.i.d. Bernoulli random sequences with $\mathbb{P}\left(\nu_{k}=1\right)=\nu$, $\mathbb{P}\left(\gamma_{k}=1\right)=\gamma$, and $\mathbb{P}\left(\tau_{k}=1\right)=\tau$. They describe the packet 
losses in the controller-to-actuator (C/A) channel, the sensorto-estimator (S/E) channel, and the ACK channel, respectively. That is,

- $\gamma_{k}=1$ stands for that the observation $y_{k}$ has been successfully received by estimator, otherwise $\gamma_{k}=0$.

- $\nu_{k}=1$ indicates that the control input $u_{k}$ has been successfully delivered to the actuator, otherwise $\nu_{k}=0$.

- $\tau_{k}=1$ means that the ACK signal, i.e., the value of $\nu_{k}$, has been successfully delivered to the estimator; Otherwise, $\tau_{k}=0$ and $\nu_{k}$ is unavailable for the estimator.

For the system described in (1), some standard assumptions are made as follows.

Assumption 1: The pair $\left(A, Q^{1 / 2}\right)$ is controllable, and the pair $(A, C)$ is observable. The initial state $x_{0} \sim \mathcal{N}_{x_{0}}\left(\bar{x}_{0}, P_{0}\right)$, and $x_{0}, \omega_{k}, v_{k}, \nu_{k}, \gamma_{k}$, and $\tau_{k}$ are mutually independent.

\section{B. Problems formulation}

Define the information set $\mathcal{I}_{k} \triangleq\left\{\mathrm{y}^{k}, \gamma^{k}, \tau^{k}\right\}$ with $\mathcal{G}_{0} \triangleq \phi$ (empty set), where $\mathrm{y}^{k} \triangleq\left\{y_{k}, \cdots, y_{1}\right\}, \gamma^{k} \triangleq\left\{\gamma_{k}, \cdots, \gamma_{1}\right\}$, and $\tau^{k} \triangleq\left\{\tau_{k}, \cdots, \tau_{0}\right\}$. In this paper, we study the following four problems.

Problem 1: (Optimal estimation) Determine the optimal state estimation, denoted by $\hat{x}_{k}$, in the minimum mean square error (MMSE) sense. That is, to find $\hat{x}_{k}$, minimizing $\mathbb{E}\left[\| x_{k}-\right.$ $\left.\hat{x}_{k} \mid \mathcal{I}_{k} \|^{2}\right]$.

The optimal LQG problem is formulated as follows. Given a integer $N$, let $\left\{W_{k}\right\}$ and $\left\{\Lambda_{k}\right\}$ for $1 \leq k \leq N$ be positive definite matrices. Define the cost functions as follows:

$$
\begin{aligned}
J_{N}\left(\pi_{N-1}, \bar{x}_{0}, P_{0}\right)= & \mathbb{E}\left[x_{N}^{\prime} W_{N} x_{N}+\sum_{k=0}^{N-1} x_{k}^{\prime} W_{k} x_{k}\right. \\
& \left.+\nu_{k} u_{k}^{\prime} \Lambda_{k} u_{k} \mid \pi_{N-1}, \bar{x}_{0}, P_{0}\right]
\end{aligned}
$$

where $\pi_{N-1}=\left\{u_{0}, \ldots, u_{N-1}\right\}$ is a sequence of the control inputs, and $u_{k}$ is a function of $\mathcal{I}_{k}$, i.e., $u_{k}=f_{k}\left(\mathcal{I}_{k}\right)$.

Assumption 2: The pair $(A, B)$ is stabilizable, and the pair $\left(A, W^{1 / 2}\right)$ is detectable.

Problem 2: (Optimal LQG control) Determine the optimal control sequence, denoted by $\pi_{N-1}^{*}$, that minimizes the cost function $J_{N}$, i.e.,

$$
J_{N}^{*}=J_{N}\left(\pi_{N-1}^{*}, \bar{x}_{0}, P_{0}\right)=\min _{\pi_{N-1}} J_{N}\left(\pi_{N-1}, \bar{x}_{0}, P_{0}\right) .
$$

As we will see later, based on the optimal estimator, it is difficult, sometimes impossible, to solve the optimal LQG problem. Thus, we consider the following sub-optimal LQG problem.

Problem 3: (Sub-optimal LQG control) Whether does there exist a sub-optimal linear estimator, based on which the LQG controller can be obtained?

An important property for a contrller is that whether or not it can stablize the closed-loop system. Since the Quasi-TCPlike system is a stochastic system, the stability is considered in the mean sense as follows:

Definition 1: The closed-loop system is said to be mean square stable, if both $\mathbb{E}\left[\left\|x_{k}\right\|^{2}\right]$ and $\mathbb{E}\left[\left\|\hat{x}_{k}\right\|^{2}\right]$ are bounded.

Problem 4: (Mean square stability) Determine the condition under which the closed-loop system is mean square stable.

The answers to Problems $1-4$ are presented in Theorems $1-4$, respectively.

\section{THE OPTIMAL ESTIMATOR AND SOLVABILITY OF THE OPTIMAL LQG CONTROLLER}

\section{A. Optimal estimator}

It is well known in [33] that the desired optimal estimation $\hat{x}_{k}$ is given by $\mathbb{E}\left[x_{k} \mid \mathcal{I}_{k}\right]$. Thus, we first derive the pdf of $x_{k}$ in Lemma 3 , and then compute the optimal estimation in Theorem 1 . Let $\bar{x}_{k} \triangleq \mathbb{E}\left[x_{k} \mid \mathcal{I}_{k-1}\right]$ denote the state prediction, and let $\bar{P}_{k}$ and $P_{k}$ denote the prediction and estimation error covariances, respectively.

It is shown later that the pdf of $x_{k}$ is a Gaussian mixture. Two uesful results on Gaussian and Gaussian mixture pdfs are formulated in the following two lemmas.

Lemma 1: [34, pp. 44] Given two independent random variables $X \sim \mathcal{N}_{X}(m, P)$ and $Z \sim \mathcal{N}_{Z}(0, W)$. Let $Y=C X+Z$ where $C$ is a constant matrix. Then

$$
\begin{aligned}
P_{Y} & =C P C^{\prime}+W \\
p(Y) & =\mathcal{N}_{Y}\left(C m, C P C^{\prime}+W\right) \\
p(X \mid Y) & =\mathcal{N}_{X}(m+K(y-C m),(I-K C) P)
\end{aligned}
$$

where $K=P C^{\prime}\left(C P C^{\prime}+W\right)^{-1}$, and $P_{Y}$ is the covariance of $Y$.

Lemma 2: [33, pp. 213] Consider the following discretetime linear system:

$$
\begin{aligned}
& x_{k}=A x_{k-1}+B_{k-1} u_{k-1}+\omega_{k-1} \\
& y_{k}=C x_{k}+v_{k}
\end{aligned}
$$

where the $B_{k}$ is a time-varying deterministic parameter. $\omega_{k} \sim$ $\mathcal{N}(0, Q)$ and $v_{k} \sim \mathcal{N}(0, R)$ are mutually independent. Then the following facts hold.

(i) If $p\left(x_{k-1}\right)=\sum_{i=1}^{N} \alpha_{k-1}^{[i]} \mathcal{N}_{x_{k-1}}\left(m_{k-1}^{[i]}, M_{k-1}\right)$, then the time-update pdf

$$
p\left(x_{k}\right)=\sum_{i=1}^{N} \bar{\alpha}_{k}^{[i]} \mathcal{N}_{x_{k}}\left(\bar{m}_{k}^{[i]}, \bar{M}_{k}\right),
$$

where $\bar{\alpha}_{k}^{[i]}=\alpha_{k-1}^{[i]}, \bar{m}_{k}^{[i]}=A m_{k-1}^{[i]}+B_{k-1} u_{k-1}$, and $\bar{M}_{k}=A M_{k-1} A^{\prime}+Q$.

(ii) If $p\left(x_{k}\right)$ takes the form as in (3), then the measurementupdate pdf

$$
p\left(x_{k} \mid y_{k}\right)=\sum_{i=1}^{N} \alpha_{k}^{[i]} \mathcal{N}_{x_{k}}\left(m_{k}^{[i]}, M_{k}\right),
$$

where $m_{k}^{[i]}=\bar{m}_{k}^{[i]}+K_{k}\left(y_{k}-C \bar{m}_{k}^{[i]}\right), K_{k}=$ $\bar{M}_{k} C^{\prime}\left(C \bar{M}_{k} C^{\prime}+R\right)^{-1}, M_{k}=\left(I-K_{k} C\right) \bar{M}_{k}, \alpha_{k}^{[i]}=$ $\bar{\alpha}_{k}^{[i]} \phi_{k}^{[i]} / c, \phi_{k}^{[i]}=\mathcal{N}_{y_{k}}\left(C \bar{m}_{k}^{[i]}, P_{k}^{Y}\right), P_{k}^{Y}=C \bar{M}_{k} C^{\prime}+R$, and $c=\sum_{j=1}^{N} \bar{\alpha}_{k}^{[j]} \phi_{k}^{[j]}$.

(iii) Denote $\hat{x}_{k}=\mathbb{E}\left[x_{k} \mid y_{k}\right]$ and $P_{k}=\mathbb{E}\left[\left(x_{k}-\hat{x}_{k}\right)_{I}^{2} \mid y_{k}\right]$. If $p\left(x_{k} \mid y_{k}\right)$ takes the form as in (4), then

$$
\hat{x}_{k}=\sum_{i=1}^{N} \alpha_{k}^{[i]} m_{k}^{[i]}, P_{k}=B+\sum_{i=1}^{N} \alpha_{k}^{[i]}\left(m_{k}^{[i]}-\hat{x}_{k}\right)_{I}^{2} .
$$

1) Probability density function of $x_{k}$ : Denote by $n_{k}$ the number of the lost ACK signals during time 0 to $k-1$.

Lemma 3: Let $N_{k}=2^{n_{k}}$. For $1 \leq i \leq N_{k}$,

$$
\begin{aligned}
p\left(x_{k} \mid \mathcal{I}_{k-1}\right) & =\sum_{i=1}^{N_{k}} \bar{\alpha}_{k}^{[i]} \mathcal{N}_{x_{k}}\left(\bar{m}_{k}^{[i]}, \bar{M}_{k}\right) \\
p\left(x_{k} \mid \mathcal{I}_{k}\right) & =\sum_{i=1}^{N_{k}} \alpha_{k}^{[i]} \mathcal{N}_{x_{k}}\left(m_{k}^{[i]}, M_{k}\right),
\end{aligned}
$$




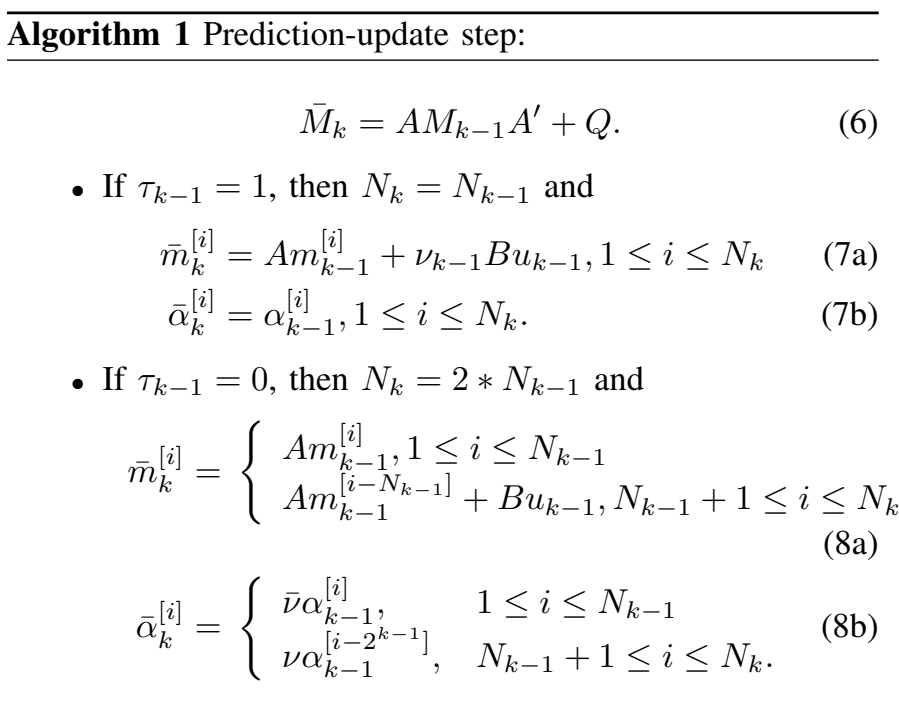

where $\bar{\nu} \triangleq 1-\nu$.

Algorithm 2 Measurement-update step:
$\begin{aligned} & \text { For } 1 \leq i \leq N_{k} \\ & m_{k}^{[i]}=\bar{m}_{k}^{[i]}+\gamma_{k} K_{k}\left(y_{k}-C \bar{m}_{k}^{[i]}\right)\end{aligned}$
where
\[ \begin{array}{l}K_{k}=\bar{M}_{k} C^{\prime}\left(C \bar{M}_{k} C^{\prime}+R\right)^{-1} \\ M_{k}=\bar{M}_{k}-\gamma_{k} K_{k} C \bar{M}_{k}\end{array} \]
and $\alpha_{k}^{[i]}=\left(\frac{\phi_{k}^{[i]}}{\sum_{j=1}^{N_{k}} \phi_{k}^{[j]} \bar{\alpha}_{k}^{[j]}}\right)^{\gamma_{k}} \bar{\alpha}_{k}^{[i]}$
where $\phi_{k}^{[i]} \triangleq \mathcal{N}_{y_{k}}\left(C \bar{m}_{k}^{[i]}, P_{k}^{Y}\right)$ and $P_{k}^{Y} \triangleq C \bar{M}_{k} C^{\prime}+R$

where $\left\{\bar{\alpha}_{k}^{[i]}, \alpha_{k}^{[i]}\right\},\left\{\bar{m}_{k}^{[i]}, m_{k}^{[i]}\right\}$, and $\left\{\bar{M}_{k}, M_{k}\right\}$ are computed by Algorithms 1 and 2 with $\alpha_{0}^{[1]}=1, m_{0}^{[1]}=\bar{x}_{0}$, and $M_{0}=$ $P_{0}$.

Proof: We prove this lemma by mathematical induction.

Step 1: Consider the case $k=1$. Then $x_{1}=A x_{0}+\nu_{0} B u_{0}+\omega_{0}$.

- If $\tau_{0}=1$, then the value of $\nu_{0}$ is known and $n_{1}=0$. From (2b) in Lemma 1 , it follows that $p\left(x_{1}\right)=\mathcal{N}_{x_{1}}\left(\bar{x}_{1}, \bar{P}_{1}\right)$, where $\bar{x}_{1}=A \bar{x}_{0}$ and $\bar{P}_{1}=A P_{0} A^{\prime}+Q$. By computing (6) and (7) with $k=1$, we can obtain $\bar{\alpha}_{1}^{[1]}, \bar{m}_{1}^{[1]}$, and $\bar{M}_{1}$. Substituting them into (5a) yields $p\left(x_{1}\right)=\mathcal{N}_{x_{1}}\left(\bar{x}_{1}, \bar{P}_{1}\right)$. Thus, (5a), (6), and (7) hold for $k=1$ and $\tau_{0}=1$.

- If $\tau_{0}=0$, then the value of $\nu_{0}$ is unknown. Then $n_{1}=1$ and $N_{1}=2$. By the total probability law, we have

$$
\begin{aligned}
p\left(x_{1}\right) & =p\left(x_{1} \mid\left\{\nu_{0}=0\right\}\right) p\left(\left\{\nu_{0}=0\right\}\right) \\
& +p\left(x_{1} \mid\left\{\nu_{0}=1\right\}\right) p\left(\left\{\nu_{0}=1\right\}\right)
\end{aligned}
$$

In $p\left(x_{1} \mid\left\{\nu_{0}=0\right\}\right), \nu_{0}$ takes the value 0 and is a deterministic quantity. By (2b), $p\left(x_{1} \mid\left\{\nu_{0}=0\right\}\right)=\mathcal{N}_{x_{1}}\left(A \bar{x}_{0}, \bar{M}_{1}\right)$ where $\bar{M}_{1}=A P_{0} A^{\prime}+Q$. Similarly, by using (2b) again, $p\left(x_{1} \mid\left\{\nu_{0}=1\right\}\right)=\mathcal{N}_{x_{1}}\left(A \bar{x}_{0}+B u_{0}, \bar{M}_{1}\right)$. If we set $\bar{\alpha}_{1}^{[1]}=\bar{\nu}, \bar{\alpha}_{1}^{[2]}=\nu, \bar{m}_{1}^{[1]}=A \bar{x}_{0}$, and $\bar{m}_{1}^{[2]}=A \bar{x}_{0}+B u_{0}$, then (12) can be rewritten as

$$
p\left(x_{1}\right)=\bar{\alpha}_{1}^{[1]} \mathcal{N}_{x_{1}}\left(\bar{m}_{1}^{[1]}, \bar{M}_{1}\right)+\bar{\alpha}_{1}^{[2]} \mathcal{N}_{x_{1}}\left(\bar{m}_{1}^{[2]}, \bar{M}_{1}\right) .
$$

It is easy to verify that $p\left(x_{1}\right)$ computed by (5a), (6), and (8) with $k=1$ is equal to (13). Hence, (5a), (6), and (8) hold for $k=1$ and $\tau_{0}=0$.

Consequently, (5a), (6), (7), and (8) hold for $k=1$.

Step 2: In Step 1, we have proved that (5a) holds at $k=1$, that is,

$$
p\left(x_{1}\right)=\sum_{i=1}^{N_{1}} \bar{\alpha}_{1}^{[i]} \mathcal{N}_{x_{1}}\left(\bar{m}_{k}^{[i]}, \bar{M}_{1}\right) .
$$

- If $\gamma_{1}=0$, there is no observation $y_{1}$ and thus $p\left(x_{1} \mid \mathcal{I}_{1}\right)=$ $p\left(x_{1}\right)$. Let $p\left(x_{1} \mid \mathcal{I}_{1}\right)$ take the form

$$
p\left(x_{1} \mid \mathcal{I}_{1}\right)=\sum_{i=1}^{2^{N_{1}}} \alpha_{1}^{[i]} \mathcal{N}_{x_{1}}\left(m_{1}^{[i]}, M_{1}\right) .
$$

It is evident that $\alpha_{1}^{[i]}=\bar{\alpha}_{1}^{[i]}, m_{1}^{[i]}=\bar{m}_{1}^{[i]}$, and $M_{1}=\bar{M}_{1}$, since $p\left(x_{1} \mid \mathcal{I}_{1}\right)=p\left(x_{1}\right)$. Hence, (5b), (9), (10), and (11) hold at $k=1$ and $\gamma_{1}=0$.

- If $\gamma_{1}=1$, with the observation $y_{1}, p\left(x_{1} \mid \mathcal{I}_{1}\right)$ can be derectly obtained from $p\left(x_{1}\right)$ in (14) by using Lemma 2 (ii). We still let $p\left(x_{1} \mid y_{1}\right)$ take the form as in (15). It is easy to check that $p\left(x_{1} \mid \mathcal{I}_{1}\right)$ and the parameters $\left\{\alpha_{1}^{[i]}, m_{1}^{[i]}, M_{1}\right\}$, obtained from $p\left(x_{1}\right)$ in (14) by using Lemma 2 (ii), are completely indentical to those computed by (5b), (9)-(11) at $k=1$ and $\gamma_{1}=1$.

From Steps 1 and 2, it follows that (5)-(11) hold at $k=1$. Suppose that (5)-(11) hold for $1, \ldots, n$. We check the case $k=n+1$ as follows.

Step 3: For $k=n+1, x_{n+1}=A x_{n}+\nu_{n} B u_{n}+\omega_{n}$.

- If $\tau_{n}=1$, then the value of $\nu_{n}$ is known and $n_{n+1}=$ $n_{n} \cdot p\left(x_{n+1} \mid \mathcal{I}_{n}\right)$ can be obtained from $p\left(x_{n} \mid \mathcal{I}_{n}\right)$ by using Lemma 2 (i). It is easy to verify that the $p\left(x_{n+1} \mid \mathcal{I}_{n}\right)$ obtained is equal to the $p\left(x_{n+1} \mid \mathcal{I}_{n}\right)$ computed by (5a), (6), and (7) with $k=n+1$. Thus, (5a), (6), and (7) hold at $k=n+1$ and $\tau_{n}=1$.

- If $\tau_{n}=0$, then the value of $\nu_{n}$ is unknown to the estimator, $n_{n+1}=n_{n}+1$, and $N_{n+1}=2 N_{n}$. By using the total probability law,

$$
\begin{aligned}
p\left(x_{n+1} \mid \mathcal{I}_{n}\right) & =p\left(x_{n+1} \mid \mathcal{I}_{n},\left\{\nu_{n}=0\right\}\right) p\left(\left\{\nu_{n}=0\right\}\right) \\
& +p\left(x_{n+1} \mid \mathcal{I}_{n},\left\{\nu_{n}=1\right\}\right) p\left(\left\{\nu_{n}=1\right\}\right) .
\end{aligned}
$$

By applying Lemma 2 (i) to $p\left(x_{n+1} \mid \mathcal{I}_{n},\left\{\nu_{n}=0\right\}\right)$ and $p\left(x_{n+1} \mid \mathcal{I}_{n},\left\{\nu_{n}=1\right\}\right)$, we have

$$
\begin{aligned}
& p\left(x_{n+1} \mid \mathcal{I}_{n},\left\{\nu_{n}=0\right\}\right) \\
= & \sum_{i=1}^{2^{n_{n}}} \alpha_{n}^{[i]} \mathcal{N}_{x_{n+1}}\left(\bar{m}_{n+1}^{[i]}, \bar{M}_{n+1}\right)
\end{aligned}
$$

where $\bar{m}_{n+1}^{[i]}=A m_{n}^{[i]}$ and $\bar{M}_{n+1}=A M_{n} A^{\prime}+Q$, for $1 \leq i \leq 2^{n_{n}}$;

$$
\begin{aligned}
& p\left(x_{n+1} \mid \mathcal{I}_{n},\left\{\nu_{n}=1\right\}\right) \\
= & \sum_{i=1}^{2^{n_{n}}} \alpha_{n}^{[i]} \mathcal{N}_{x_{n+1}}\left(\bar{m}_{n+1}^{[i]}, \bar{M}_{n+1}\right)
\end{aligned}
$$

where $\bar{m}_{n+1}^{[i]}=A m_{n}^{[i]}+B u_{n}$, for $1 \leq i \leq 2^{n_{n}}$. By substituting (17) and (18) into (16), $p\left(x_{n+1} \mid \mathcal{I}_{n}\right)$ can be rewritten as: $p\left(x_{n+1} \mid \mathcal{I}_{n}\right)=$ $\sum_{i=1}^{2^{n} n+1} \bar{\alpha}_{n+1}^{[i]} \mathcal{N}_{x_{n+1}}\left(\bar{m}_{n+1}^{[i]}, \bar{M}_{n+1}\right) \quad$ where $\left\{\bar{m}_{n+1}^{[i]}\right.$, 
$\left.\bar{\alpha}_{n+1}^{[i]}, \bar{M}_{n+1}\right\}$ are equal to (6) and (8) with $k=n+1$, which means that (5a), (6), and (8) hold for $k=n+1$ and $\tau_{n}=0$.

Step 4: By using Lemma 2 (ii) and following the same line of argument in Step 2, it is easy to verify that (5b), (9), (10), and (11) hold at $k=n+1$. For the save of space, the proof is not presented here.

From Steps 3 and 4, it follows that (5)-(11) hold at $k=$ $n+1$, which completes the proof.

2) Optimal estimator for the Quasi-TCP-like system:

Theorem 1 (Optimal estimator): The optimal estimator for the Quasi-TCP-like system is the following:

$$
\begin{aligned}
\hat{x}_{k} & =\sum_{i=1}^{2^{n_{k}}} \alpha_{k}^{[i]} m_{k}^{[i]} \\
P_{k} & =M_{k}+\sum_{i=1}^{2^{n_{k}}} \alpha_{k}^{[i]}\left(m_{k}^{[i]}-\hat{x}_{k}\right)_{I}^{2}
\end{aligned}
$$

where $\alpha_{k}^{[i]}, m_{k}^{[i]}$, and $M_{k}$ can be computed by Algorithms 1 and 2.

Proof: Since $p\left(x_{k} \mid \mathcal{I}_{k}\right)$ in (5a) is a Gaussian mixture, (19) can be readily obtained by applying Lemma 2 (iii) to (5a).

Remark 1: The impacts of the random loss of ACK are formulated as follows:

- It is known in [17] that $M_{k}$ in (10), in fact, is the estimation error covariances for the TCP-like system. Hence, the summation part in (19b) can be viewed as the degradation of estimation performance caused by the random losses of ACK signals.

- From Algorithms 1 and 2, it follows that the number of the terms in the Gaussian mixture pdfs (5) doubles at the time when the ACK signal is lost. As time passes, the number of the terms will exponentially increases. Consequently, the computation of $\hat{x}_{k}$ and $P_{k}$ requires exponentially increasing time, and eventually exhausts computer's memory. Thus, in general, the optimal estimation cannot be used in practice.

\section{B. Solvability of the Optimal LQG problem}

For the UDP- or Quasi-TCP-like system, it is only known that it is difficult to obtain the LMMSE-estimator-based LQG control [17, 23], whereas it is little known about the optimal solution. In this section, by an example we reveal the difficulties in solving the optimal LQG problem, and then make a conclusion on its solvability in Theorem 2 .

We define the optimal value function $V_{k}\left(x_{k}\right)$ as follows and use the cost-to-go dynamic programming approach to derive the optimal control.

$$
\begin{aligned}
V_{N}\left(x_{N}\right)= & \mathbb{E}\left[x_{N}^{\prime} W_{N} x_{N} \mid \mathcal{I}_{N}\right] \\
V_{k}\left(x_{k}\right)= & \min _{u_{k}} \mathbb{E}\left[x_{k}^{\prime} W_{k} x_{k}\right. \\
& \left.+\nu_{k} u_{k}^{\prime} \Lambda_{k} u_{k}+V_{k+1}\left(x_{k+1}\right) \mid \mathcal{I}_{k}\right] .
\end{aligned}
$$

Example 1: Consider a simple scalar system [17] with $A=$ $B=C=1, W_{N}=W_{k}=1, \Lambda_{k}=0, R=1$. We further assume that there is no system noise (i.e., $\omega_{k} \equiv 0$ and $Q=0$ ) and no observation packet losses (i.e., $\gamma_{k} \equiv 1$ ). Without loss of generality, we suppose that $\tau_{N-1}=0$ and $\tau_{N-2}=1$.
1) Calculations of $V_{N}\left(x_{N}\right), V_{N-1}\left(x_{N-1}\right)$, and $V_{N-2}\left(x_{N-2}\right)$ : $V_{N}\left(x_{N}\right)=\mathbb{E}\left[x_{N}^{\prime} W_{N} x_{N} \mid \mathcal{I}_{N}\right]=\mathbb{E}\left[x_{N}^{2} \mid \mathcal{I}_{N}\right]$.

$$
\begin{aligned}
& V_{N-1}\left(x_{N-1}\right)=\min _{u_{N-1}} \mathbb{E}\left[x_{N-1}^{2}+V_{N}\left(x_{N}\right) \mid \mathcal{I}_{N-1}\right] \\
= & \min _{u_{N-1}} \mathbb{E}\left[x_{N-1}^{2}+\left(x_{N-1}+\nu_{N-1} u_{N-1}\right)^{2} \mid \mathcal{I}_{N-1}\right] \\
= & \min _{u_{N-1}} \mathbb{E}\left[2 x_{N-1}^{2} \mid \mathcal{I}_{N-1}\right]+\nu u_{N-1}^{2}+2 \nu u_{N-1} \hat{x}_{N-1} .
\end{aligned}
$$

By solving $\partial V_{N-1}\left(x_{N-1}\right) / \partial u_{N-1}=0$, the optimal control is obtained as $u_{N-1}^{*}=-\hat{x}_{N-1} \cdot V_{N-1}\left(x_{N-1}\right)=$ $\mathbb{E}\left[2 x_{N-1}^{2} \mid \mathcal{I}_{N-1}\right]-\nu \hat{x}_{N-1}^{2}=\mathbb{E}\left[(2-\nu) x_{N-1}^{2} \mid \mathcal{I}_{N-1}\right]+$ $\nu P_{N-1}$.

By virtue of $\tau_{N-2}=1, \nu_{N-2}$ is known to the estimator and controller.

$$
\begin{aligned}
& V_{N-2}\left(x_{N-2}\right)=\min _{u_{N-2}} \mathbb{E}\left[x_{N-2}^{2}+V_{N-1}\left(x_{N-1}\right) \mid \mathcal{I}_{N-2}\right] \\
= & \min _{u_{N-2}} \mathbb{E}\left[x_{N-2}^{2}+(2-\nu)\left(x_{N-2}+\nu_{N-2} u_{N-2}\right)^{2}\right. \\
& \left.+\nu P_{N-1} \mid \mathcal{I}_{N-2}\right] \\
= & \min _{u_{N-2}} \mathbb{E}\left[(3-\nu) x_{N-2}^{2} \mid \mathcal{I}_{N-2}\right]+2 \nu_{N-2}(2-\nu) \hat{x}_{N-2} u_{N-2} \\
& +\nu_{N-2}(2-\nu) u_{N-2}^{2}+\nu \mathbb{E}\left[P_{N-1} \mid \mathcal{I}_{N-2}\right] .
\end{aligned}
$$

To obtain $V_{N-2}$, we proceed to compute $P_{N-1}$ and $\mathbb{E}\left[P_{N-1} \mid \mathcal{I}_{N-2}\right]$.

2) Computation of $P_{N-1}$ : Note that $\gamma_{k} \equiv 1, \tau_{N-1}=0$.

From (8b), we have $\bar{\alpha}_{N-1}^{[i]}=\alpha_{N-2}^{[i]}$. Let $c \triangleq$ $\sum_{i=1}^{2^{n} N-1} \alpha_{N-2}^{[i]} \phi_{N-1}^{[i]}$. From (9), we have for $1 \leq i \leq 2^{k}$,

$$
\begin{aligned}
\alpha_{N-1}^{[i]} & =\frac{1}{c} \phi_{N-1}^{[i]} \alpha_{N-2}^{[i]} \\
m_{N-1}^{[i]} & =\mathbb{A}_{N-1} m_{N-2}^{[i]}+\Gamma_{A},
\end{aligned}
$$

where $\Gamma_{A} \triangleq \nu_{N-2} \mathbb{K}_{N-2} B u_{N-2}+K_{N-1} y_{N-1}$.

From (19a) and (22a),

$$
\hat{x}_{N-1}=\sum_{i=1}^{2^{n_{N-1}}} \alpha_{N-1}^{[i]} m_{N-1}^{[i]}=\mathbb{A}_{N-1} x_{N-2}^{*}+\Gamma_{A},
$$

where $x_{N-2}^{*} \triangleq \frac{1}{c} \sum_{i=1}^{2^{n} N-1} \phi_{N-1}^{[i]} \alpha_{N-2}^{[i]} m_{N-2}^{[i]}$.

By (19b), (22a) and (23), we have

$$
\begin{aligned}
P_{N-1} & =M_{N-1}+\sum_{i=1}^{2^{n} N-1} \alpha_{N-2}^{[i]}\left(m_{N-2}^{[i]}-\hat{x}_{N-2}\right)^{2} \\
& =M_{N-1}+\mathbb{A}_{N-1} \Gamma_{B},
\end{aligned}
$$

where $\Gamma_{B} \triangleq \sum_{i=1}^{2^{n} N-1} \frac{1}{c} \phi_{N-1}^{[i]} \alpha_{N-2}^{[i]}\left(m_{N-2}^{[i]}-x_{N-2}^{*}\right)^{2}$.

$$
\begin{aligned}
\Gamma_{B}= & \sum_{i=1}^{2^{n_{N-1}}} \frac{1}{c} \phi_{N-1}^{[i]} \alpha_{N-2}^{[i]}\left(\left(m_{N-2}^{[i]}\right)^{2}-2 m_{N-2}^{[i]} x_{N-2}^{*}\right. \\
& \left.+\left(x_{N-2}^{*}\right)^{2}\right) \\
= & \left(x_{N-2}^{*}\right)^{2}+\sum_{i=1}^{2^{n_{N-1}}} \frac{1}{c} \phi_{N-1}^{[i]} \alpha_{N-2}^{[i]}\left(m_{N-2}^{[i]}\right)^{2} \\
& -\sum_{i=1}^{2^{n_{N-1}}} 2 \frac{1}{c} \phi_{N-1}^{[i]} \alpha_{N-2}^{[i]} m_{N-2}^{[i]} x_{N-2}^{*} \\
= & -\left(x_{N-2}^{*}\right)^{2}+\sum_{i=1}^{2^{n_{N-1}}} \frac{1}{c} \phi_{N-1}^{[i]} \alpha_{N-2}^{[i]}\left(m_{N-2}^{[i]}\right)^{2}
\end{aligned}
$$

3) Computation of $\mathbb{E}\left[P_{N-1} \mid \mathcal{I}_{N-2}\right]$ :

Since $y_{k}=C x_{k}+v_{k}, p\left(y_{k} \mid \mathcal{I}_{k-1}\right)$ can be obtained from $p\left(x_{k} \mid \mathcal{I}_{k-1}\right)$ by applying Lemma 2 (i) to (5a). That is,

$$
\begin{aligned}
p\left(y_{k} \mid \mathcal{I}_{k-1}\right) & =\sum_{i=1}^{2^{n_{k}}} \mathcal{N}_{y_{k}}\left(C \bar{m}_{k}^{[i]}, P_{k}^{Y}\right) \bar{\alpha}_{k}^{[i]} \\
& =\sum_{i=1}^{2^{n_{k}}} \phi_{k}^{[i]} \bar{\alpha}_{k}^{[i]} .
\end{aligned}
$$


Then we have $p\left(y_{N-2} \mid \mathcal{I}_{N-2}\right)=c$ due to $\bar{\alpha}_{N-1}^{[i]}=\alpha_{N-2}^{[i]}$. Note that $\mathcal{I}_{N-2}=\left\{y_{N-2}, \ldots, y_{1}, \tau_{N-2}, \ldots, \tau_{0}\right\}$. From (11) and (19b), it is clear that $P_{N-1}$ contains $y_{N-1}$. Thus

$$
\begin{aligned}
\mathbb{E}\left[P_{N-1} \mid \mathcal{I}_{N-2}\right] & =\int_{-\infty}^{\infty} P_{N-1} p\left(y_{N-1} \mid \mathcal{I}_{N-2}\right) \mathrm{d} y_{N-1} \\
& =\int_{-\infty}^{\infty}\left(\Gamma_{C}+\Gamma_{D}\right) \mathrm{d} y_{N-1},
\end{aligned}
$$

where

$$
\begin{gathered}
\Gamma_{C}=-c\left(x_{N-2}^{*}\right)^{2}=\frac{\left(\sum_{i=1}^{2^{n_{N-1}}} \alpha_{N-2}^{[i]} \phi_{N-1}^{[i]} m_{N-2}^{[i]}\right)^{2}}{\sum_{i=1}^{2^{n}-1} \alpha_{N-2}^{[i]} \phi_{N-1}^{[i]}} \\
\Gamma_{D}=c M_{N-1}+\mathbb{A}_{N-1} \sum_{i=1}^{2^{n_{N-1}} \phi_{N-1}^{[i]} \alpha_{N-2}^{[i]}\left(m_{N-2}^{[i]}\right)^{2} .}
\end{gathered}
$$

Since $c$ is a Gaussian mixture function and $\phi_{N-1}^{[i]}$ is a Gaussian function, there is a analytic expression for $\int_{-\infty}^{\infty} \Gamma_{D} \mathrm{~d} y_{N-1}$

4) Three difficulties are presented as follows:

- The Gaussian function $\phi_{N-1}^{[i]}$ occurs in both the numerator and denominator of $\Gamma_{C}$. From the knowledge of calculus, it is clear that there is no analytic expression for $\int_{-\infty}^{\infty} \Gamma_{C} \mathrm{~d} y_{N-1}$, even when $n_{N-1}=1$. Moreover, with the random losses of ACK signals, the number of $\phi_{N-1}^{[i]}$ will exponentially increase, which further confirms the fact that there is no analytic expression for $\int_{-\infty}^{\infty} \Gamma_{C} \mathrm{~d} y_{N-1}$ and $\mathbb{E}\left[P_{N-1} \mid \mathcal{I}_{N-2}\right]$ either.

- Note that the desired optimal $u_{N-1}^{*}$ minimizing $V_{N-2}(N-2)$ in (21) is in fact a function not a deterministic quantity. Without analytic expression for $\mathbb{E}\left[P_{N-1} \mid \mathcal{I}_{N-2}\right]$, the nonlinear optimization cannot be further performed to obtain the optimal control $u_{N-1}^{*}$ in $V(N-2)$;

- The number of the Gaussian functions $\phi_{N-1}^{[i]}$ in $\Gamma_{C}$ will exponentially increase, making its computation time-consuming. Meanwhile, in solving the LQG problem, the optimal estimation $\hat{x}_{k}$ is required, and its computation also is time-consuming.

For this simplified system, these three difficulties are enough to prevent the optimal LQG problem from being solved.

Theorem 2 (Solvability of the optimal LQG problem): For the general Quasi-TCP-like system, it is impossible to solve the optimal LQG problem.

Proof: It is clear that for the general Quasi-TCP-like system, these three difficulties mentioned above still exist. Both the calculation of $\int_{-\infty}^{\infty} \Gamma_{C} \mathrm{~d} y_{N-1}$ and the aforementioned nonlinear optimization are technically difficult. More importantly, due to the random losses of ACK, the time for the computation will tend to infinity, and computer's memory will be eventually exhausted. Therefore, we claim that it is impossible to solve the optimal LQG problem.

Remark 2: The conclusion in Theorem 2 is also applicable to the UDP-like system, since the ACK in the UDP-like system is completely lost.

\section{SUB-OPTIMAL LQG CONTROL}

The conclusion in Theorem 2 motivates us to develop a sub-optimal but efficient solution to the estimation and LQG problems for the Quasi-TCP-like system. In the following, we first develop a sub-optimal linear estimator, and then based on it we derive the LQG controller. Finally, we establish the conditions for the mean square stability of the closed-loop system.

\section{A. Sub-optimal Linear Estimator}

The structure of the optimal estimator is complex, but the $K_{k}$ in (10a) can be recursively calculated. By this $K_{k}$, we design a sub-optimal linear estimator as in Algorithm 3.

In Algorithm 3, the symbols $\bar{x}_{k}$ and $\hat{x}_{k}$ are recycled to denote the predicted and estimated system states for this suboptimal estimator, respectively. Then $\bar{P}_{k}$ and $P_{k}$ are recycled to denote the corresponding prediction and estimation error covariances, respectively. Define a function

$$
g(\gamma, M)=A M A^{\prime}-\gamma A M C^{\prime}\left(C M C^{\prime}+R\right)^{-1} C M A^{\prime}+Q .
$$

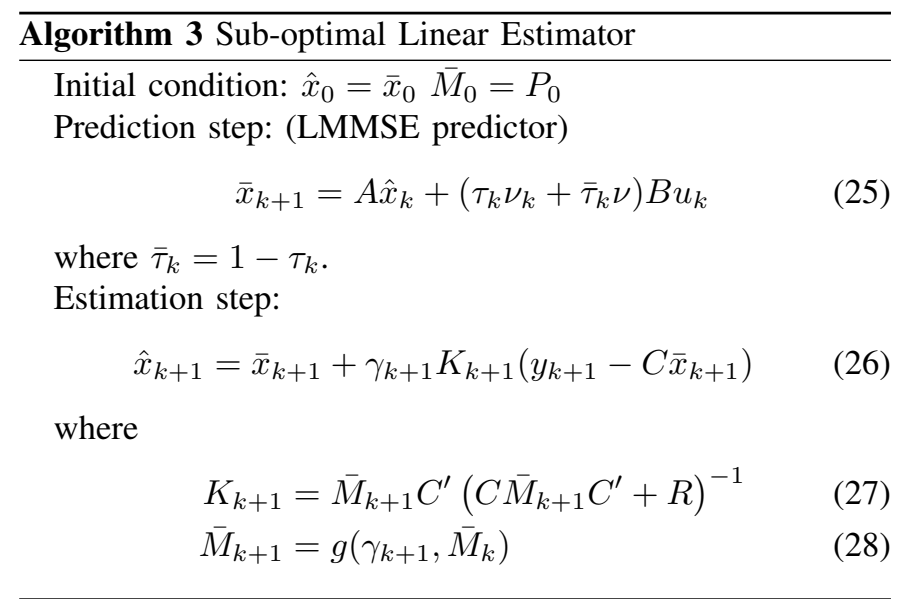

Remark 3: In Algorithm 3, we use the LMMSE preditor to obtain the predicted system state. For the estimation step, the estimator gain $K_{k}$ in (27) is in fact the $K_{k}$ in (10a) computed by the $\bar{M}_{k}$, which occurs in each term of the Gaussian mixture pdf (5a). The way to design this $K_{k}$ is inspired by [35]. In [35], by constructing auxiliary system states, a sub-optimal estimator was developed for the UDP-like without observation packet loss. The resulting estimator gain is identical to the one computed by the $\bar{M}_{k}$ in each term of the Gaussian mixture pdf. The benefit of this design method is that the $K_{k}$ is not a nonlinear function of $u_{k}$, and thus the nonlinear optimization problem is circumvented.

Remark 4: Technically speaking, the estimation performance of the sub-optimal estimator in Algorithm 3 is inferior to that of the LMMSE estimator, but in the backgound of the LQG problem they are quite close, which will be shown and explained later in Section V.

Lemma 4: The prediction and estimation error covariances can be calculated as follows:

$$
\begin{aligned}
\bar{P}_{k+1}= & A P_{k} A^{\prime}+Q+\bar{\tau}_{k} \bar{\nu} \nu B u_{k} u_{k}^{\prime} B^{\prime} \\
P_{k+1}= & \left(I-\gamma_{k+1} K_{k+1} C\right) \bar{P}_{k+1}\left(I-\gamma_{k+1} K_{k+1} C\right)^{\prime} \\
& +\gamma_{k+1} K_{k+1} R K_{k+1}^{\prime} .
\end{aligned}
$$

Proof: From (1a) and (25), we have

$$
x_{k+1}-\bar{x}_{k+1}=A\left(x_{k}-\hat{x}_{k}\right)+\bar{\tau}_{k}\left(\nu_{k}-\nu\right) B u_{k}+\omega_{k} .
$$


If $\tau_{k}=1$, then $\bar{\tau}_{k}=0$. By applying (2a) to (31), we obtain the covariance of $x_{k+1}-\bar{x}_{k+1}$, i.e., $\bar{P}_{k+1}=A P_{k} A^{\prime}+Q$. If $\tau_{k}=$ 0 , then $\bar{\tau}_{k}=1$ and $\nu_{k}$ is an unknown random quantity. By using (2a) again, we have $\bar{P}_{k+1}=A P_{k} A^{\prime}+Q+\bar{\nu} \nu B u_{k} u_{k}^{\prime} B^{\prime}$. Therefore, (29) holds for $\tau_{k}=1$ and 0.

From (1a) and (26), we have

$$
\begin{aligned}
& x_{k+1}-\hat{x}_{k+1} \\
= & x_{k+1}-\bar{x}_{k+1}-\gamma_{k+1} K_{k+1}\left(C x_{k+1}+v_{k+1}-C \bar{x}_{k+1}\right) \\
= & \left(I-\gamma_{k+1} K_{k+1} C\right)\left(x_{k+1}-\bar{x}_{k+1}\right)-\gamma_{k+1} K_{k+1} v_{k+1} .
\end{aligned}
$$

The value of $\gamma_{k+1}$ is known for the estimator. By applying (2a) to (32) and noting that $\gamma_{k+1}^{2}=\gamma_{k+1}$, it is easy to verify that (30) holds. The proof is completed.

\section{B. LQG control}

To derive the finite horizon LQG controller, we first calculate $\mathbb{E}\left[\operatorname{tr}\left(H P_{k+1}\right) \mid \mathcal{I}_{k}\right]$ in Lemma 5. Such quantity is required in Lemma 6 to derive the optimal value function $V_{k}\left(x_{k}\right)$.

Define $\mathbb{K}_{k} \triangleq I-K_{k} C$.

Lemma 5: Given a matrix $H$, let $T \triangleq \gamma \mathbb{K}_{k+1}^{\prime} H \mathbb{K}_{k+1}+\bar{\gamma} H$. Then

$$
\begin{aligned}
& \mathbb{E}\left[\operatorname{tr}\left(H P_{k+1}\right) \mid \mathcal{I}_{k}\right]=\operatorname{tr}\left(A^{\prime} T A P_{k}\right)+\operatorname{tr}(T Q) \\
& +\bar{\tau} \bar{\nu} \nu \operatorname{tr}\left(u_{k}^{\prime} B^{\prime} T B u_{k}\right)+\gamma \operatorname{tr}\left(K_{k+1}^{\prime} H K_{k+1} R\right) .
\end{aligned}
$$

Proof: By substituting (29) into (30) and then taking mathematical expectation to $P_{k+1}$, we have

$$
\begin{gathered}
\mathbb{E}\left[P_{k+1} \mid \mathcal{I}_{k}\right]=\gamma \mathbb{K}_{k+1}\left(A P_{k} A^{\prime}+Q+\bar{\tau} \bar{\nu} \nu B u_{k} u_{k}^{\prime} B^{\prime}\right) \mathbb{K}_{k+1}^{\prime} \\
+\gamma K_{k+1} R K_{k+1}^{\prime}+\bar{\gamma}\left(A P_{k} A^{\prime}+Q+\bar{\tau} \bar{\nu} \nu B u_{k} u_{k}^{\prime} B^{\prime}\right) .
\end{gathered}
$$

By using the property that $\mathbb{E}[\operatorname{tr}(A)]=\operatorname{tr}(\mathbb{E}[A])$ and $\operatorname{tr}(B A P A)=\operatorname{tr}(A B A P)$,

$$
\begin{aligned}
& \mathbb{E}\left[\operatorname{tr}\left(H P_{k+1}\right) \mid \mathcal{I}_{k}\right]=\operatorname{tr}\left(H \mathbb{E}\left[P_{k+1} \mid \mathcal{I}_{k}\right]\right) \\
= & \operatorname{tr}\left(A^{\prime}\left(\gamma \mathbb{K}_{k+1}^{\prime} H \mathbb{K}_{k+1}+\bar{\gamma} H\right) A P_{k}\right) \\
& +\operatorname{tr}\left(\left(\gamma \mathbb{K}_{k+1}^{\prime} H \mathbb{K}_{k+1}+\bar{\gamma} H\right) Q\right) \\
& +\bar{\tau} \bar{\nu} \nu \operatorname{tr}\left(u_{k}^{\prime} B^{\prime}\left(\gamma \mathbb{K}_{k+1}^{\prime} H \mathbb{K}_{k+1}+\bar{\gamma} H\right) B u_{k}\right) \\
& +\gamma \operatorname{tr}\left(K_{k+1}^{\prime} H K_{k+1} R\right) \\
= & \operatorname{tr}\left(A^{\prime} T A P_{k}\right)+\operatorname{tr}(T Q)+\bar{\tau} \bar{\nu} \nu \operatorname{tr}\left(u_{k}^{\prime} B^{\prime} T B u_{k}\right) \\
& +\gamma \operatorname{tr}\left(K_{k+1}^{\prime} H K_{k+1} R\right) .
\end{aligned}
$$

The proof is completed.

In the following, we use the cost-to-go dynamic programming approach to obtain the $V_{k}\left(x_{k}\right)$.

Lemma 6: Based on the sub-optimal estimator in Algorithm $3, V_{k}\left(x_{k}\right)$ defined in (20) can be calculated as follows:

$$
V_{k}\left(x_{k}\right)=\mathbb{E}\left[x_{k}^{\prime} Z_{k} x_{k} \mid \mathcal{I}_{k}\right]+\operatorname{tr}\left(H_{k} P_{k}\right)+\Delta_{k}
$$

where $K_{k}$ is computed by (27) and (28), and

$$
\begin{aligned}
L_{k}= & -\left(\Lambda_{k}+B^{\prime}\left(Z_{k+1}+\bar{\tau} \bar{\nu} T_{k+1}\right) B\right)^{-1} B^{\prime} Z_{k+1} A \\
T_{k+1}= & \gamma \mathbb{K}_{k+1}^{\prime} H_{k+1} \mathbb{K}_{k+1}+\bar{\gamma} H_{k+1} \\
Z_{k}= & A^{\prime} Z_{k+1} A+W_{k}-\nu A^{\prime} Z_{k+1} B\left(\Lambda_{k}+\right. \\
& \left.B^{\prime}\left(Z_{k+1}+\bar{\tau} \bar{\nu} T_{k+1}\right) B\right)^{-1} B^{\prime} Z_{k+1} A \\
H_{k}= & A^{\prime} T_{k+1} A+W_{k}+A^{\prime} Z_{k+1} A-Z_{k}
\end{aligned}
$$

$$
\begin{aligned}
\Delta_{k}= & \Delta_{k+1}+\operatorname{tr}\left(T_{k+1} Q\right)+\operatorname{tr}\left(Z_{k+1} Q\right) \\
& +\operatorname{tr}\left(\left(K_{k+1}^{\prime} H_{k+1} K_{k+1}\right) R\right)
\end{aligned}
$$

with $Z_{N}=W_{N}, H_{N}=0$, and $\Delta_{N}=0$.

Proof: We prove this lemma by mathematical induction. It is evident that (33) holds at time $N$. Suppose that (33) holds for $N, \cdots, k+1$. Now we check $V_{k}\left(x_{k}\right)$.

$$
\begin{aligned}
& V_{k}\left(x_{k}\right) \\
= & \min _{u_{k}} \mathbb{E}\left[x_{k}^{\prime} W_{k} x_{k}+\nu_{k} u_{k}^{\prime} \Lambda_{k} u_{k}+V_{k+1}\left(x_{k+1}\right) \mid \mathcal{I}_{k}\right] \\
= & \min _{u_{k}} \mathbb{E}\left[x_{k}^{\prime}\left(W_{k}+A^{\prime} Z_{k+1} A\right) x_{k}+\omega_{k+1}^{\prime} Z_{k+1} \omega_{k+1}\right. \\
& +\nu_{k} u_{k}^{\prime}\left(\Lambda_{k}+B^{\prime} Z_{k+1} B\right) u_{k}+2 \nu_{k} u_{k}^{\prime} B^{\prime} Z_{k+1} A x_{k} \\
& \left.+\operatorname{tr}\left(H_{k+1} P_{k+1}\right)+\Delta_{k+1} \mid \mathcal{I}_{k}\right] \\
\stackrel{(a)}{=} & \min _{u_{k}} \mathbb{E}\left[x_{k}^{\prime}\left(W_{k}+A^{\prime} Z_{k+1} A\right) x_{k} \mid \mathcal{I}_{k}\right]+\operatorname{tr}\left(A^{\prime} T_{k+1} A P_{k}\right) \\
& +\left\{\Delta_{k+1}+\operatorname{tr}\left(Z_{k+1} Q\right)+\operatorname{tr}\left(T_{k+1} Q\right)\right. \\
& \left.+\gamma \operatorname{tr}\left(K_{k+1}^{\prime} H_{k+1} K_{k+1} R\right)\right\} \\
& +\nu \bar{\nu} \bar{\tau} u_{k}^{\prime} B^{\prime} T_{k+1} B u_{k} \\
& +\nu u_{k}^{\prime}\left(\Lambda_{k}+B^{\prime} Z_{k+1} B\right) u_{k}+2 \nu u_{k}^{\prime} B^{\prime} Z_{k+1} A \hat{x}_{k}
\end{aligned}
$$

where $\stackrel{(a)}{=}$ is obtained by using Lemma 5 . Then we solve $\partial V_{k}\left(x_{k}\right) / \partial u_{k}=0$ and get the $u_{k}$ which minimizes $V_{k}\left(x_{k}\right)$ as follows

$$
u_{k}=-\left(\Lambda_{k}+B^{\prime}\left(Z_{k+1}+\bar{\tau} \bar{\nu} T_{k+1}\right) B\right)^{-1} B^{\prime} Z_{k+1} A \hat{x}_{k} .
$$

The quantities in $\{\cdot\}$ of (35) are equal to the $\Delta_{k}$ in $(34 \mathrm{e})$. Substituting this $u_{k}$ back into (35) yields

$$
\begin{aligned}
& V_{k}\left(x_{k}\right) \\
= & \mathbb{E}\left[x_{k}^{\prime}\left(W_{k}+A^{\prime} Z_{k+1} A\right) x_{k} \mid \mathcal{I}_{k}\right]+\Delta_{k}+\operatorname{tr}\left(A^{\prime} T_{k+1} A P_{k}\right) \\
& -\nu \hat{x}_{k}^{\prime} A^{\prime} Z_{k+1} B\left(\Lambda_{k}+B^{\prime}\left(Z_{k+1}+\bar{\tau} \bar{\nu} T_{k+1}\right) B\right)^{-1} B^{\prime} Z_{k+1} A \hat{x}_{k} \\
\stackrel{(a)}{=} \mathbb{E}\left[x_{k}^{\prime} Z_{k} x_{k} \mid \mathcal{I}_{k}\right]+\Delta_{k}+\operatorname{tr}\left(A^{\prime} T_{k+1} A P_{k}\right) & +\operatorname{tr}\left(\left(W_{k}+A^{\prime} Z_{k+1} A-Z_{k}\right) P_{k}\right) \\
& +\stackrel{(b)}{=} \mathbb{E}\left[x_{k}^{\prime} Z_{k} x_{k} \mid \mathcal{I}_{k}\right]+\Delta_{k}+\operatorname{tr}\left(H_{k} P_{k}\right),
\end{aligned}
$$

where $\stackrel{(a)}{=}$ is obtained by using $(34 \mathrm{c})$ and the existing result ([17], Lemma 4.1) that $\mathbb{E}\left[x_{k}^{\prime} S x_{k}\right]=\hat{x}_{k}^{\prime} S \hat{x}_{k}+\operatorname{tr}\left(S P_{k}\right)$. The equality $\stackrel{(b)}{=}$ is obtained by using (34d). Hence, (33) holds for the time $k$. The proof is completed.

Based on Lemma 6, the results on the LQG control problem are formulated in the following theorem.

Theorem 3 (LQG control): For the Quasi-TCP-like system, based on the sub-optimal estimator in Algorithm 3,

- the finite horizon LQG controller is $u_{k}=L_{k} \hat{x}_{k}$, and the corresponding cost function

$$
\begin{aligned}
& J_{N}=x_{0}^{\prime} Z_{0} x_{0}+\operatorname{tr}\left(\left(Z_{0}+H_{0}\right) P_{0}\right) \\
& +\sum_{k=1}^{N} \operatorname{tr}\left(\left(T_{k}+Z_{k}\right) Q+\left(K_{k}^{\prime} H_{k} K_{k}\right) R\right),
\end{aligned}
$$

where $L_{k}, Z_{k}, T_{k}, H_{k}$ are computed by (34), and $\hat{x}_{k}$ and $K_{k}$ are computed by Algorithm 3.

- There is no solution to the infinite horizon LQG problem.

Proof: From the dynamic programming approach, it follows that the control sequence $u_{k}=L_{k} \hat{x}_{k}$ obtained in 
Lemma 6 is the desired optimal control that minimizes the cost function $J_{N}$, and $J_{N}=V_{0}\left(x_{0}\right)$. From (33) and (34), it is easy to obtain $J_{N}$ as in (36).

Similar to the TCP-like system, the estimator gain $K_{k}$ is a random quantity, making $\frac{1}{k} J_{k}$ and $L_{k}$ unconvergent. Therefore, there is no solution to the infinite horizon LQG problem. The proof is completed.

Remark 5: (Separation principle) From Algorithm 3, it is known that the estimator gain $K_{k}$ is independent of the design of the LQG controller. However, the LQG controller depends on $K_{k}$, and the estimation error covariance $P_{k}$ depends on the control inputs. Consequently, the separation principle does not hold for the Quasi-TCP-like system.

\section{Stability of the closed-loop systems}

In the sequel, we show that under some conditions the controller $u_{k}=L_{k} \hat{x}_{k}$ can stabilize the closed-loop QuasiTCP-like system, where $L_{k}$ is computed by (34).

To study the stability of the closed-loop systems, we let $W_{k}=W, \Lambda_{k}=\Lambda$, and $G_{k} \triangleq H_{k}+Z_{k}$. Note that $S=g(1, S)$ is the standard algebraic Riccati equation, where $g(\cdot)$ defined in (24). It is well known that under Assumption 1, there is an unique positive definite solution $S_{\infty}$ for $S_{\infty}=g\left(1, S_{\infty}\right)$. For the convenience of formulation, we define some symbols. Denote the maximum sigular value of $\mathbb{K}$ by $\lambda_{\mathbb{K}}$, where $\mathbb{K}=$ $I-S_{\infty} C^{\prime}\left(C S_{\infty} C^{\prime}+R\right)^{-1} C$. Define $\eta \triangleq \gamma\left(\lambda_{\mathbb{K}}\right)^{2}+\bar{\gamma}$ and $\rho \triangleq \bar{\tau} \bar{\nu} \eta$.

Three condtions are given as follows: Condtion 1: $B$ is square and invertible; Condtion 2: $C$ is full column rank; and Condtion 3: $P_{0} \geq S_{\infty}$.

Theorem 4 (Stability of the closed-loop systems): Consider the system in (1) with the LQG controller $u_{k}=L_{k} \hat{x}_{k}$ where $L_{k}$ is computed by (34).

(i) If $Z_{k}$ and $G_{k}$ are bounded, then the system is mean square stable.

(ii) If Conditions 1,2 , and 3 are satified, then a sufficient condition for the boundedness of $Z_{k}$ and $G_{k}$ is

$$
\lambda_{A}^{2}(\eta+\nu-2 \eta \nu)<(\eta+\nu-\eta \nu) .
$$

Proof of part (i): Let $\mathcal{K}_{k}=\left(I-\gamma_{k} K_{k} C\right)$. We start with calculating $x_{k}$ and $e_{k}$. By substituting $u_{k}=L \hat{x}_{k}$ into (1) and using $e_{k}=x_{k}-\hat{x}_{k}$,

$$
\begin{aligned}
x_{k+1} & =A x_{k}+\nu_{k} B u_{k}+\omega_{k} \\
& =\left(A+\nu_{k} B L_{k+1}\right) x_{k}-\nu_{k} B L_{k+1} e_{k}+\omega_{k} .
\end{aligned}
$$

By combining (31) and (32), we have

$$
\begin{aligned}
e_{k+1}= & x_{k+1}-\hat{x}_{k+1} \\
= & \left.\mathcal{K}_{k+1}\left(A e_{k}+\bar{\tau}_{k}\left(\nu_{k}-\nu\right) B L_{k+1}\right) \hat{x}_{k}+\omega_{k}\right) \\
& -\gamma_{k+1} K_{k+1} v_{k+1} \\
= & \bar{\tau}_{k}\left(\nu_{k}-\nu\right) \mathcal{K}_{k+1} B L_{k+1} x_{k} \\
& +\mathcal{K}_{k+1}\left(A-\bar{\tau}_{k}\left(\nu_{k}-\nu\right) B L_{k+1}\right) e_{k} \\
& +\mathcal{K}_{k+1} \omega_{k}-\gamma_{k+1} K_{k+1} v_{k+1} .
\end{aligned}
$$

The homogenous parts of (37) and (38) are the following:

$$
x_{k+1}=\left(A+\nu_{k} B L_{k+1}\right) x_{k}-\nu_{k} B L_{k+1} e_{k}
$$

$$
\begin{aligned}
e_{k+1}= & \bar{\tau}_{k}\left(\nu_{k}+\nu\right) \mathcal{K}_{k+1} B L_{k+1} x_{k} \\
& +\mathcal{K}_{k+1}\left(A-\bar{\tau}_{k}\left(\nu_{k}+\nu\right) B L_{k+1}\right) e_{k} .
\end{aligned}
$$

Since $\mathbb{E}\left[\left\|\omega_{k}\right\|^{2}\right]=\operatorname{tr}(Q)$ and $\mathbb{E}\left[\left\|v_{k+1}\right\|^{2}\right]=\operatorname{tr}(R)$ in (37) and (38) are bounded, it was pointed out in [28] that if the homogenous parts of (37) and (38) are asymptotically stable, then the system equations (37) and (38) are mean square stable.

To study the asympotic stability of (39) and (40), we follow the similar line of augument developed in [28], which requires the calculation of $x_{k}^{\prime} Z_{k} x_{k}+e_{k}^{\prime} H_{k} e_{k}$. However, it would be tedious to compute this quantity directly via (39) and (40), which can be seen in [28]. Actually, majorities of the derivations for computing this quantity have been performed in calculating $V_{k}\left(x_{k}\right)$ in Lemma 6. Therefore, in the following we employ the results on $V_{k}\left(x_{k}\right)$ to compute this quantity.

Denote the optimal control by $u_{k}^{*}$. From (20), we have

$$
V_{k}\left(x_{k}\right)=\mathbb{E}\left[x_{k}^{\prime} W x_{k}+\nu_{k}\left(u_{k}^{*}\right)^{\prime} \Lambda u_{k}^{*}+V_{k+1}\left(x_{k+1}\right) \mid \mathcal{I}_{k}\right] .
$$

According to the definition of the mean square stability, it is the $\mathbb{E}\left[\left\|x_{k}\right\|^{2}\right]$ not the $\mathbb{E}\left[\left\|x_{k}\right\|^{2} \mid \mathcal{I}_{k}\right]$ that is considered. Thus, taking mathematical expectation with respect to $\mathcal{I}_{k}$ yields

$$
\mathbb{E}\left[V_{k+1}\left(x_{k+1}\right)-V_{k}\left(x_{k}\right)\right]=-\mathbb{E}\left[x_{k}^{\prime} W x_{k}+\nu\left(u_{k}^{*}\right)^{\prime} \Lambda u_{k}^{*}\right] .
$$

From (33) and by noting that $\mathbb{E}\left[e_{k}^{\prime} H_{k} e_{k}\right]=\operatorname{tr}\left(H_{k} P_{k}\right)$, we obtain

$$
\mathbb{E}\left[V_{k}\left(x_{k}\right)\right]=\mathbb{E}\left[x_{k}^{\prime} Z_{k} x_{k}+e_{k}^{\prime} H_{k} e_{k}\right]+\mathbb{E}\left[\Delta_{k}\right] .
$$

Then

$$
\begin{aligned}
& \mathbb{E}\left[x_{k+1}^{\prime} Z_{k+1} x_{k+1}+e_{k+1}^{\prime} H_{k+1} e_{k+1}-\left(x_{k}^{\prime} Z_{k} x_{k}+e_{k}^{\prime} H_{k} e_{k}\right)\right] \\
= & \mathbb{E}\left[V_{k+1}\left(x_{k+1}\right)-\Delta_{k+1}-\left(V_{k}\left(x_{k}\right)-\Delta_{k}\right)\right] \\
= & -\mathbb{E}\left[x_{k}^{\prime} W x_{k}+\nu\left(u_{k}^{*}\right)^{\prime} \Lambda u_{k}^{*}\right]+\left(\operatorname{tr}\left(T_{k+1} Q\right)\right. \\
& \left.+\operatorname{tr}\left(Z_{k+1} Q\right)+\operatorname{tr}\left(\left(K_{k+1}^{\prime} H_{k+1} K_{k+1}\right) R\right)\right)
\end{aligned}
$$

where the last equality is obtained by (41) and (34e).

In Lemma $6, x_{k}$ and $e_{k}$ are determined by (37) and (38). While what we consider is their homogenous parts, i.e., (41) and (42), in which there is no noise, which is equivalent to letting $Q=R=0$ in $V_{k}\left(x_{k}\right)$. Therefore, for the homogenous parts (41) and (42), by letting $Q=R=0$ in (43),

$$
\begin{aligned}
& \mathbb{E}\left[x_{k+1}^{\prime} Z_{k+1} x_{k+1}+e_{k+1}^{\prime} H_{k+1} e_{k+1}-\left(x_{k}^{\prime} Z_{k} x_{k}+e_{k}^{\prime} H_{k} e_{k}\right)\right] \\
& =-\mathbb{E}\left[x_{k}^{\prime} W x_{k}+\nu_{k}\left(u_{k}^{*}\right)^{\prime} \Lambda u_{k}^{*}\right] .
\end{aligned}
$$

Summing up this equality for $k=0$ to $n-1$ yields

$$
\begin{aligned}
& \mathbb{E}\left[x_{n}^{\prime} Z_{n} x_{n}+e_{n}^{\prime} H_{n} e_{n}-\left(x_{0}^{\prime} Z_{0} x_{0}+e_{0}^{\prime} H_{0} e_{0}\right)\right] \\
= & -\sum_{k=0}^{n-1} \mathbb{E}\left[x_{k}^{\prime} W x_{k}+\nu\left(u_{k}^{*}\right)^{\prime} \Lambda u_{k}^{*}\right] .
\end{aligned}
$$

Due to $\mathbb{E}\left[x_{n}^{\prime} Z_{n} x_{n}+e_{n}^{\prime} H_{n} e_{n}\right] \geq 0$, we have $\mathbb{E}\left[x_{0}^{\prime} Z_{0} x_{0}+\right.$ $\left.e_{0}^{\prime} H_{0} e_{0}\right] \geq \sum_{k=0}^{n-1} \mathbb{E}\left[x_{k}^{\prime} W x_{k}+\nu\left(u_{k}^{*}\right)^{\prime} \Lambda u_{k}^{*}\right]$. By the hypothesis that $\left\{Z_{k}\right.$ and $\left.G_{k}\right\}$ are bounded, we have $\bar{Z} \geq Z_{0}$ and $\bar{G} \geq G_{0}=Z_{0}+H_{0} \geq H_{0}$. Then $\mathbb{E}\left[x_{0}^{\prime} \bar{Z} x_{0}+e_{0}^{\prime} \bar{G} e_{0}\right] \geq$ $\sum_{k=0}^{n-1} \mathbb{E}\left[x_{k}^{\prime} W x_{k}+\nu\left(u_{k}^{*}\right)^{\prime} \Lambda u_{k}^{*}\right]$. The boundedness of the series $\sum_{k=0}^{n=1} \mathbb{E}\left[x_{k}^{\prime} W x_{k}\right]$ implies $\lim _{k \rightarrow \infty} \mathbb{E}\left[x_{k}^{\prime} W x_{k}\right]=0$. Due to $W>0, \mathbb{E}\left[x_{k}^{\prime} x_{k}\right]=\mathbb{E}\left[\left\|x_{k}\right\|^{2}\right] \rightarrow 0$. Since $\mathbb{E}\left[x_{k}^{\prime} W x_{k}\right]=$ $\hat{x}_{k}^{\prime} W \hat{x}_{k}+\mathbb{E}\left[e_{k}^{\prime} W e_{k}\right]$, we have $\lim _{k \rightarrow \infty} \mathbb{E}\left[\hat{x}_{k}^{\prime} W \hat{x}_{k}\right]=0$, i.e., $\mathbb{E}\left[\left\|\hat{x}_{k}\right\|^{2}\right] \rightarrow 0$, which implies the asympotic stability of (39) 
and (40). Hence, (37) and (38) are mean square stable. The proof of part (i) is completed.

After introducing some preliminaries and lemmas as follows, we continue the proof of part (ii).

To study the boundedness of $Z_{k}$ and $G_{k}$, we reverse the time index in (34) and then rewrite (34) as follows:

$$
\begin{aligned}
L_{k+1} & =-\left(\Lambda+B^{\prime}\left(Z_{k}+\bar{\tau} \bar{\nu} T_{k}\right) B\right)^{-1} B^{\prime} Z_{k} A \\
Z_{k+1} & =\Phi_{X}\left(Z_{k}, Z_{k}+\bar{\tau} \bar{\nu} T_{k}\right) \\
G_{k+1} & =A^{\prime}\left(\gamma \mathbb{K}_{k}^{\prime} H_{k} \mathbb{K}_{k}+\bar{\gamma} H_{k}+Z_{k}\right) A+W \\
\Delta_{k+1} & =\Delta_{k}+\operatorname{tr}\left(T_{k} Q+\left(K_{k}^{\prime} H_{k} K_{k}\right) R+Z_{k} Q\right)
\end{aligned}
$$

with $Z_{0}=W$ and $H_{0}=0$, where

$$
\Phi_{X}(Z, Y) \triangleq A^{\prime} Z A+W-\nu A^{\prime} Z B\left(\Lambda+B^{\prime} Y B\right)^{-1} B^{\prime} Z A .
$$

Define two operators as follows:

$$
\begin{aligned}
\Phi_{Z}(Z, G, \rho) \triangleq & \Phi_{X}(Z,(1-\rho) Z+\rho G) \\
= & A^{\prime} Z A+W-\nu A^{\prime} Z B(\Lambda \\
& \left.+B^{\prime}((1-\rho) Z+\rho G) B\right)^{-1} B^{\prime} Z A \\
\Phi_{G}(Z, G, \eta) \triangleq & (1-\eta) A^{\prime} G A+\eta A^{\prime} Z A+W .
\end{aligned}
$$

Lemma 7: Some results on $g(1, X), \Phi_{X}, \Phi_{Z}$, and $\Phi_{G}$ are formulated as follows ([17, pp. 182] and [36, Theorems 10.6 and 10.7]):

(i) $g(1, X), \Phi_{X}, \Phi_{Z}$, and $\Phi_{G}$ are monotonically increasing functions. Namely, if $Z_{1} \geq Z_{2}$ and $Y_{1} \geq Y_{2}$, then $g\left(1, Z_{1}\right) \geq g\left(1, Z_{2}\right), \Phi_{X}\left(Z_{1}, Y_{1}\right) \geq \Phi_{X}\left(Z_{2}, Y_{2}\right)$, $\Phi_{Z}\left(Z_{1}, Y_{1}, \rho\right) \geq \Phi_{Z}\left(Z_{2}, Y_{2}, \rho\right)$, and $\Phi_{G}\left(Z_{1}, Y_{1}, \eta\right) \geq$ $\Phi_{G}\left(Z_{2}, Y_{2}, \eta\right)$.

(ii) If Condition 1 is satisfied, then a necessary and sufficient condition for the convergences of $Z_{k+1}=$ $\Phi_{Z}\left(Z_{k}, G_{k}, \rho\right)$ and $G_{k+1}=\Phi_{G}\left(Z_{k}, G_{k}, \eta\right)$ is $\lambda_{A}^{2}(\eta+$ $\nu-2 \eta \nu)<(\eta+\nu-\eta \nu)$.

(iii) If $S_{0} \geq S_{\infty}$, then $S_{0} \geq S_{k} \geq S_{\infty}$.

Lemma 8: Let $X>0$ and $Y \geq 0$, and $C$ is a matrix with compatible dimension. Then

(i) ([37], Theorem 7.7.3 and Corollary 7.7.4)

The following three inequalities are equivalent: $\lambda\left(Y X^{-1}\right)<1 \Leftrightarrow X>Y \Leftrightarrow Y^{-1}>X^{-1}$.

(ii) ([38], pp. 213) The matrix inverse lemma:

$X C^{\prime}\left(C X C^{\prime}+Y\right)^{-1}=\left(X^{-1}+C^{\prime} Y^{-1} C\right)^{-1} C^{\prime} Y^{-1}$.

In the sequel, we assume that Conditions 1,2 , and 3 are satified.

Lemma 9: Let $\bar{M}_{0}=P_{0} \geq S_{\infty}$. The following facts hold.

(i) Let $S_{k+1}=g\left(1, S_{k}\right)$ with $S_{0}=\bar{M}_{0}=P_{0}$. Then $S_{\infty} \leq$ $S_{k} \leq \bar{M}_{k}$.

(ii) $F\left(\bar{M}_{k}\right)=\mathbb{K}_{k}^{\prime} H_{k} \mathbb{K}_{k}$ is monotonically decreasing, and thus $\mathbb{K}_{k}^{\prime} H_{k} \mathbb{K}_{k} \leq\left(\lambda_{\mathbb{K}}\right)^{2} H_{k}$.

Proof:

(i) We prove this lemma by mathematical induction. For $k=$ 0 , this lemma holds. Suppose that it holds for $0, \ldots, n$. We check the case $k=n+1$ as follws. By the hypothesis that $S_{n} \leq \bar{M}_{n}$ and Lemma 7 (i), $S_{n+1}=g\left(1, S_{n}\right) \leq$ $g\left(1, \bar{M}_{n}\right) \leq g\left(\gamma_{n+1}, \bar{M}_{n}\right)=\bar{M}_{n+1}$. Consequently, we have $S_{k} \leq \bar{M}_{k}$. From Lemma 7 (iii), it follows that $S_{\infty} \leq S_{k} \leq \bar{M}_{k}$. The proof is completed. (ii) Define $h(S) \triangleq\left(S^{-1}+C^{\prime} R^{-1} C\right)^{-1} C^{\prime} R^{-1} C, f(S) \triangleq$ $I-h(S)$, and $F(S) \triangleq f(S)^{\prime} H_{k} f(S)$. By Lemma 8 (ii), we have $h\left(\bar{M}_{k}\right)=K_{k} C$. Thus, $f\left(\bar{M}_{k}\right)=\mathbb{K}_{k}$ and $F\left(\bar{M}_{k}\right)=\mathbb{K}_{k}^{\prime} H_{k} \mathbb{K}_{k}$.

Suppose that $S_{1}>S_{2}$. By Lemma 8 (ii), we have $S_{1}^{-1}<S_{2}^{-1}$ and thus $S_{1}^{-1}+C^{\prime} R^{-1} C<S_{2}^{-1}+C^{\prime} R^{-1} C$. Let $Y=C^{\prime} R^{-1} C$, and $Y^{-1}$ exists by virtue of the assumption that $C$ is full column rank. By using Lemma 8 (ii) again, we have

$$
\begin{aligned}
& \left(S_{1}^{-1}+Y\right)^{-1}>\left(S_{2}^{-1}+Y\right)^{-1} \\
\stackrel{(a)}{\Rightarrow} & \lambda\left(\left(S_{2}^{-1}+Y\right)^{-1} Y Y^{-1}\left(S_{1}^{-1}+Y\right)\right)<1 \\
\Rightarrow & \lambda\left(h\left(S_{2}\right) h\left(S_{1}\right)^{-1}\right)<1 \stackrel{(b)}{\Rightarrow} h\left(S_{1}\right)>h\left(S_{2}\right) \\
\stackrel{(c)}{\Rightarrow} & f\left(S_{1}\right)<f\left(S_{2}\right) \stackrel{(d)}{\Rightarrow} \lambda\left(f\left(S_{1}\right) f\left(S_{2}\right)^{-1}\right)<1,
\end{aligned}
$$

where the inequalities on the right-hand side of $\stackrel{(a)}{\Rightarrow} \stackrel{(b)}{\Rightarrow}$, and $\stackrel{(d)}{\Rightarrow}$ are obtained by using Lemma 8 (i), and $\stackrel{(c)}{\Rightarrow}$ is obtained by noting that $f\left(S_{1}\right)<f\left(S_{2}\right)$ due to $f(S)=$ $I-h(S)$.

To compare $f\left(S_{1}\right)^{\prime} H_{k} f\left(S_{1}\right)$ with $f\left(S_{2}\right)^{\prime} H_{k} f\left(S_{2}\right)$, we consider the following inequalities.

$$
\begin{aligned}
& \lambda\left(\left(f\left(S_{1}\right) f\left(S_{2}\right)^{-1}\right)^{\prime} H_{k}\left(f\left(S_{1}\right) f\left(S_{2}\right)^{-1}\right) H_{k}^{-1}\right) \\
\leq & \lambda\left(\left(f\left(S_{1}\right) f\left(S_{2}\right)^{-1}\right)^{\prime}\right) \lambda\left(H_{k} f\left(S_{1}\right)\left(f\left(S_{2}\right)^{-1} H_{k}^{-1}\right)\right. \\
= & \left(\lambda\left(f\left(S_{1}\right) f\left(S_{2}\right)^{-1}\right)\right)^{2}<1 .
\end{aligned}
$$

From Lemma 8 (i), it follows that $\left(f\left(S_{1}\right) f\left(S_{2}\right)^{-1}\right)^{\prime} H_{k} f\left(S_{1}\right)\left(f\left(S_{2}\right)\right)^{-1}<H_{k}$, which means that $f\left(S_{1}\right)^{\prime} H_{k} f\left(S_{1}\right)<f\left(S_{2}\right)^{\prime} H_{k} f\left(S_{2}\right)$, i.e., $F\left(S_{1}\right)<F\left(S_{2}\right)$. From the result in part (i), we have $\mathbb{K}_{k}^{\prime} H_{k} \mathbb{K}_{k}=F\left(\bar{M}_{k}\right) \leq F\left(S_{\infty}\right)=\mathbb{K}^{\prime} H_{k} \mathbb{K} \leq\left(\lambda_{\mathbb{K}}\right)^{2} H_{k}$.

The proof is completed.

Lemma 10: Define $\bar{Z}_{k+1}=\Phi_{Z}\left(\bar{Z}_{k}, \bar{G}_{k}, \rho\right)$ and $\bar{G}_{k+1}=$ $\Phi_{G}\left(\bar{Z}_{k}, \bar{G}_{k}, \eta\right)$ with $\bar{Z}_{0}=Z_{0}, \bar{G}_{k}=G_{0}$. Then

$$
\bar{Z}_{k} \geq Z_{k}, \bar{G}_{k} \geq G_{k} .
$$

Proof: From (44c) and by using Lemma 9, we have

$$
\begin{aligned}
G_{k+1} & =A^{\prime}\left(\gamma \mathbb{K}_{k}^{\prime} H_{k} \mathbb{K}_{k}+\bar{\gamma} H_{k}+Z_{k}\right) A+W \\
& \leq\left(\gamma\left(\lambda_{\mathbb{K}}\right)^{2}+\bar{\gamma}\right) A^{\prime} H_{k} A+A^{\prime} Z_{k} A+W \\
& =\eta A^{\prime} G_{k} A+(1-\eta) A^{\prime} Z_{k} A+W \\
& =\Phi_{G}\left(Z_{k}, G_{k}, \rho\right) .
\end{aligned}
$$

From (34b), $Z_{k}+\bar{\tau} \bar{\nu} T_{k} \leq \bar{\tau} \bar{\nu}\left(\gamma \lambda_{\mathbb{K}}^{2}+\bar{\gamma}\right)\left(H_{k}+Z_{k}-Z_{k}\right)+Z_{k}=$ $\rho G_{k}+(1-\rho) Z_{k}$. By Lemma 7 (i),

$$
\begin{aligned}
Z_{k+1} & =\Phi_{X}\left(Z_{k}, \bar{\tau} \bar{\nu} T_{k}\right) \\
& \leq \Phi_{X}\left(Z_{k},(1-\rho) Z_{k}+\rho G_{k}\right)=\Phi_{Z}\left(Z_{k}, G_{k}, \rho\right) .
\end{aligned}
$$

We prove this lemma by mathematical induction. It is clear that (45) holds for $k=0$. Suppose that it holds for $0, \ldots, n$. We check the case $k=n+1$ as follows. From (46) (47) and Lemma 7 (i), we have $G_{n+1} \leq \Phi_{G}\left(Z_{n}, G_{n}, \rho\right) \leq$ $\Phi_{G}\left(\bar{Z}_{n}, \bar{G}_{n}, \rho\right)=\bar{G}_{n+1}$, and $Z_{n+1} \leq \Phi_{Z}\left(Z_{n}, G_{n}, \rho\right) \leq$ $\Phi_{Z}\left(\bar{Z}_{n}, \bar{G}_{n}, \rho\right)=\bar{Z}_{n+1}$. The proof is completed. 
Proof of part (ii) of Theorem 4: From Lemma 7 (ii), it follows that if Condition 1 is satisfied and the inequality $\lambda_{A}^{2}(\eta+\nu-2 \eta \nu)<(\eta+\nu-\eta \nu)$ holds, then $\bar{Z}_{k}$ and $\bar{G}_{k}$ are convergent and thus are bounded. By lemma 10, $Z_{k}$ and $G_{k}$ are bounded as well.

\section{NUMERICAL EXAMPLES}

In this section, by examples, we evaluate the performance of the proposed LQG controller and verify the main results we obtained.

\section{A. Stability of the closed-loop system:}

In the following, we present two examples to verify the mean square stability of the closed-loop system.

Example 2: Consider the unstable MIMO system in [17] with following parameters:

$$
\begin{gathered}
A=\left[\begin{array}{cccc}
1.001 & 0.005 & 0 & 0 \\
0.350 & 1.001 & -0.135 & 0 \\
-0.001 & 0 & 1.001 & 0.005 \\
-0.375 & -0.001 & 0.590 & 1.001
\end{array}\right] B=\left[\begin{array}{c}
0.001 \\
0.540 \\
-0.002 \\
-1.066
\end{array}\right] \\
C=\left[\begin{array}{cccc}
1 & 0 & 0 & 0 \\
0 & 0 & 1 & 0
\end{array}\right] R=\operatorname{diag}(0.001,0.001), Q=q q^{\prime}
\end{gathered}
$$

where $q=[0.003,1,-0.005,-2.150]^{\prime}, W=\operatorname{diag}(1,0,0,0)$, $\Lambda=2$.

Since Condistions 1 and 2 are not satisfied for this MIMO system, the boundedness of $Z_{k}$ and $G_{k}$ cannot be theoratically determined via Theorem 4 (ii). Thus, we check their boundedness by simulation. By runnig the simulation 1000 times with randomly generated $\left\{\gamma_{k}, \nu_{k}, \tau_{k}\right\}$, we found that $Z_{k}$ and $G_{k}$ are always bounded. One of these running results is shown in Fig. 2. From Theorem 4 (i), it follows that the closed-loop system is mean square stable, as shown in Fig. 2.
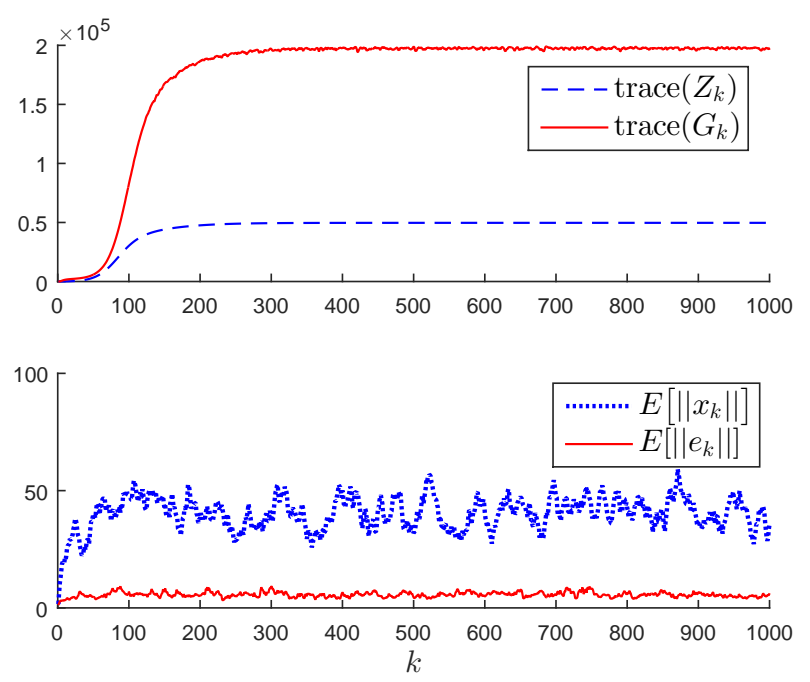

Fig. 2. The boundedness of $Z_{k}$ and $G_{k}$, and the mean square stability of the MIMO system.

Example 3: Consider the scalar unstable system used in $[19,21]$ with the parameters: $A=1.1, B=C=1, Q=$ $R=W=\Lambda=1$. Let $\gamma=\nu=\tau=0.8$. It is easy to check that for this system, Condistions 1 and 2 are satisfied, and the inequality $\lambda_{A}^{2}(\eta+\nu-2 \eta \nu)<(\eta+\nu-\eta \nu)$ holds. It follows from Theorem 4 (ii) that $Z_{k}$ and $G_{k}$ are bounded, which guarantees the mean square stability of the closed-loop system. These results are illustrated in Fig. 3.
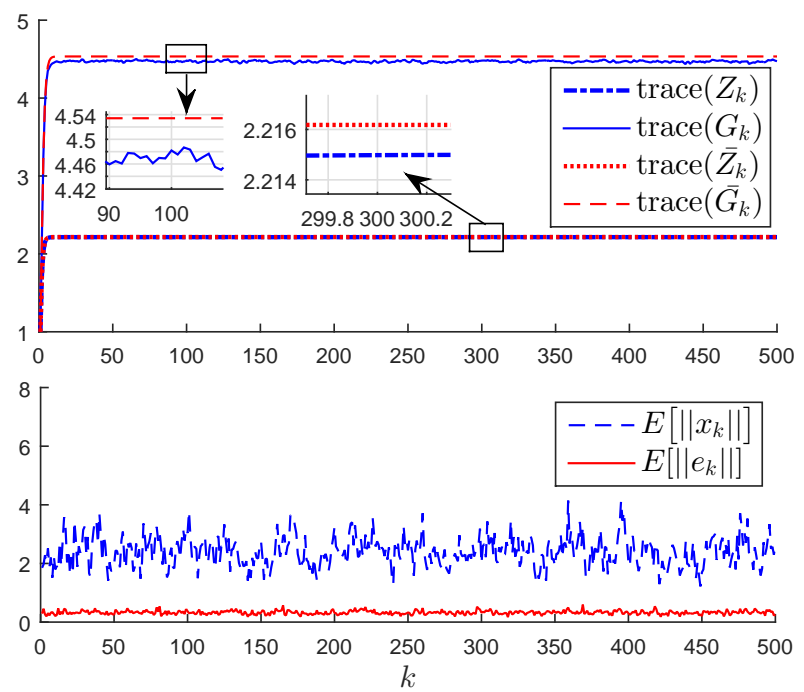

Fig. 3. The boundedness of $Z_{k}$ and $G_{k}$, and the mean square stability of the scalar system.

\section{B. Performance of the sub-optimal estimator and controller}

Example 4: To compare the estimation performance of the sub-optimal estimator we proposed with that of the LMMSE estimator, we consider the quantity $\Delta_{P}=P_{k}-P_{k}^{L}$, where $P_{k}^{L}$ denotes the estimation error covariance of the LMMSE estimator. For the scalar system above, $\Delta_{P}$ is shown in Fig.4. If the control inputs are deterministic and are independent of the estimates, like the cases $u_{k} \equiv 10$ or 20 , then the larger the magnitude of control inputs is, the more apparant the difference between their estimation performances becomes. However, it is in the background of the LQG control problem that we propose this sub-optimal estimator. Once the control inputs are determined by the LQG controller we design, the performances between these two estimators are closer, as shown in Fig. 4. The main reason is that when the system is stabilized by the LQG controller, the system states are usually near zero and then the magnitude of the feedback control is small. From (29), it is known that $\bar{P}_{k}$ is closer to the prediction error covariance of the LMMSE estimator [23]. Thus, $P_{k}$ is closer to $P_{k}^{L}$.

Example 5: For the LQG problem, $J_{k} / k$ is usually adopted to evaluate the long term performance. For the scalar (i.e., SISO) and MIMO systems presented in Examples 1 and 2, the $J_{k} / k$ is not convergent, which is shown in Fig. 5. When the ACK packet arrival rate $\tau$ is close to 1, the performance of the proposed LQG controller approches that of the optimal LQG controller for the TCP-like system (that is, the "Real TCP-like" case in Fig. 5).

\section{Conclusions}

In this paper, for the general Quasi-TCP-like systems we have proposed the optimal estimator and given a conclusion 


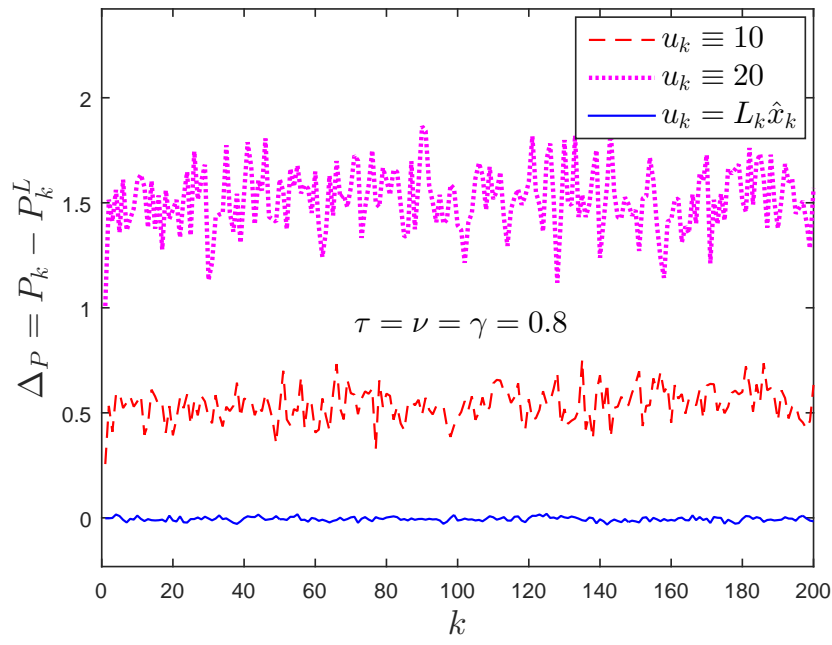

Fig. 4. The difference of the performances between the sub-optimal estimator we proposed and that of the LMMSE estimator.
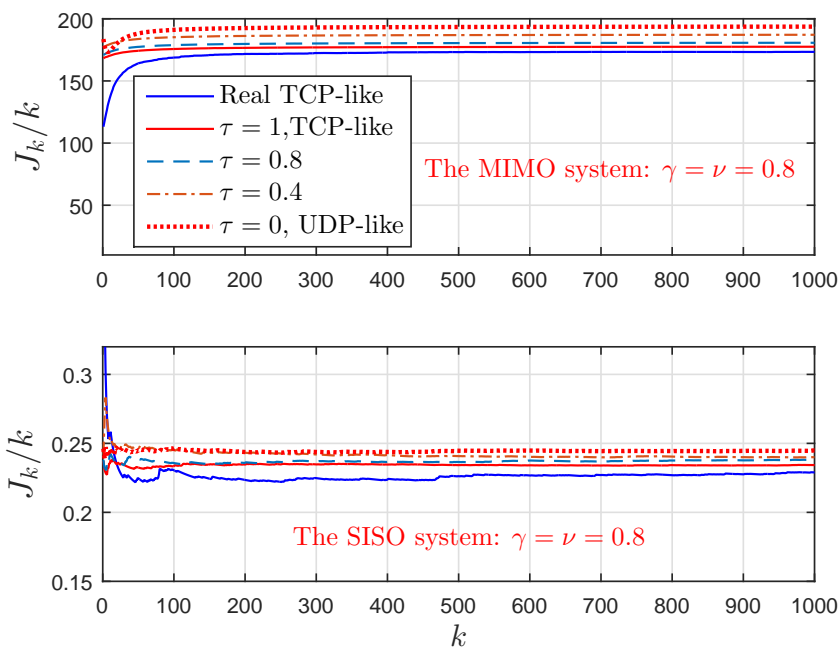

Fig. 5. The average cost function $J_{k} / k$.

on the solvability of the optimal LQG controller. Also a suboptimal LQG controller is designed. Examples are given to demonstrate the potential and effectiveness of the proposed LQG controller.

\section{REFERENCES}

[1] L. Zhang, H. Gao, and O. Kaynak, "Network-induced constraints in networked control systemsła survey," Industrial Informatics, IEEE Transactions on, vol. 9, no. 1, pp. 403-416, 2013.

[2] J. Zhang, Y. Lin, and P. Shi, "Output tracking control of networked control systems via delay compensation controllers," Automatica, vol. 57, pp. 85-92, 2015.

[3] P. Shi, H. Wang, and C.-C. Lim, "Network-based event-triggered control for singular systems with quantizations," Industrial Electronics, IEEE Transactions on, doi:10.1109/TIE.2015.2475515.

[4] R. Yang, P. Shi, and G.-P. Liu, "Filtering for discrete-time networked nonlinear systems with mixed random delays and packet dropouts," IEEE Trans. Automat. Control, vol. 56, no. 11, pp. 2655-2660, 2011.

[5] P. Shi, X. Luan, and F. Liu, " $H_{\infty}$ filtering for discrete-time systems with stochastic incomplete measurement and mixed delays," Industrial Electronics, IEEE Transactions on, vol. 59, no. 6, pp. 2732-2739, 2012

[6] L. Zhang, Y. Zhu, P. Shi, and Y. Zhao, "Resilient asynchronous $H_{\infty}$ filtering for markov jump neural networks with unideal measurements and multiplicative noises," Cybernetics, IEEE Transactions on, vol. 45, no. 12 , pp. 2840-2852, 2015.

[7] H. Yan, F. Qian, F. Yang, and H. Shi, " $H_{\infty}$ filtering for nonlinear networked systems with randomly occurring distributed delays, missing measurements and sensor saturation," Information Sciences, doi:10.1016/j.ins.2015.09.027.

[8] H. Zhang, Q. Chen, H. Yan, and J. Liu, "Robust filtering for switched stochastic system with missing measurements," Signal Processing, IEEE Transactions on, vol. 57, no. 9, pp. 3466-3474, 2009.

[9] Z. Wang, F. Yang, D. W. Ho, and X. Liu, "Robust $H_{\infty}$ control for networked systems with random packet losses," IEEE Trans. Syst., Man, Cybern., Syst.B, vol. 37, no. 4, pp. 916-924, 2007.

[10] R. Yang, P. Shi, G.-P. Liu, and H. Gao, "Network-based feedback control for systems with mixed delays based on quantization and dropout compensation," Automatica, vol. 47, no. 12, pp. 2805-2809, 2011.

[11] L. Zhang, Z. Ning, and P. Shi, "Input-output approach to control for fuzzy Markov jump systems with time-varying delays and uncertain packet dropout rate," Cybernetics, IEEE Transactions on, vol. 45, no. 11, pp. 2449-2460, 2015.

[12] L. Qiu, Y. Shi, F. Yao, G. Xu, and B. Xu, "Network-based robust $H_{2} / H_{\infty}$ control for linear systems with two-channel random packet dropouts and time delays," Cybernetics, IEEE Transactions on, vol. 45, no. 8, pp. 1450-1462, 2014.

[13] G.-P. Liu, Y. Xia, J. Chen, D. Rees, and W. Hu, "Networked predictive control of systems with random network delays in both forward and feedback channels," Industrial Electronics, IEEE Transactions on, vol. 54, no. 3, pp. 1282-1297, 2007.

[14] J. Zhang, Y. Xia, and P. Shi, "Design and stability analysis of networked predictive control systems," Control Systems Technology, IEEE Transactions on, vol. 21, no. 4, pp. 1495-1501, 2013.

[15] H. Dong, Z. Wang, J. Lam, and H. Gao, "Fuzzy-model-based robust fault detection with stochastic mixed time delays and successive packet dropouts," Systems, Man, and Cybernetics, Part B: Cybernetics, IEEE Transactions on, vol. 42, no. 2, pp. 365-376, 2012.

[16] H. Li, C. Wu, P. Shi, and Y. Gao, "Control of nonlinear networked systems with packet dropouts: interval type-2 fuzzy model-based approach," Cybernetics, IEEE Transactions on, vol. 45, no. 11, pp. 2378-2389, 2015.

[17] L. Schenato, B. Sinopoli, M. Franceschetti, K. Poolla, and S. S. Sastry, "Foundations of control and estimation over lossy networks," Proc. the IEEE, vol. 95, no. 1, pp. 163-187, 2007.

[18] H. Lin, H. Su, Z. Shu, Z.-G. Wu, and Y. Xu, "Optimal estimation in UDP-like networked control systems with intermittent inputs: Stability analysis and suboptimal filter design," IEEE Trans. Automat. Control, doi:10.1109/TAC.2015.2479195.

[19] H. Lin, H. Su, P. Shi, R. Lu, and Z.-G. Wu, "LQG control for networked control systems over packet drop links without packet acknowledgment," Journal of the Franklin Institute, vol. 352, no. 11, pp. 5042-5060, 2015.

[20] A. L. Garcia and I. Widjaja, Communication Networks. McGraw Hill, 2000.

[21] B. Sinopoli, L. Schenato, M. Franceschetti, K. Poolla, and S. Sastry, "Optimal linear LQG control over lossy networks without packet acknowledgment," Asian Journal of Control, vol. 10, no. 1, pp. 3-13, 2008.

[22] M. Kögel, R. Blind, F. Allgöwer, and R. Findeisen, "Optimal and optimal-linear control over lossy, distributed networks," in Proc. 18th IFAC World Congress, 2011, pp. $13239-13244$.

[23] E. Garone, B. Sinopoli, and A. Casavola, "LQG control over lossy TCP-like networks with probabilistic packet acknowledgements," International Journal of Systems, Control and Communications, vol. 2, no. 1, pp. 55-81, 2010.

[24] M. Moayedi, Y. K. Foo, and Y. C. Soh, "Networked LQG control over unreliable channels," International Journal of Robust and Nonlinear Control, vol. 23, no. 2, pp. 167-189, 2013.

[25] B. Sinopoli, L. Schenato, M. Franceschetti, K. Poolla, M. I. Jordan, and S. S. Sastry, "Kalman filtering with intermittent observations," IEEE Trans. Automat. Control, vol. 49, no. 9, pp. 1453-1464, 2004.

[26] K. Plarre and F. Bullo, "On Kalman filtering for detectable systems with intermittent observations," IEEE Trans. Automat. Control, vol. 54, no. 2 , pp. 386-390, 2009.

[27] Y. Mo and B. Sinopoli, "Kalman filtering with intermittent observations: tail distribution and critical value," IEEE Trans. Automat. Control, vol. 57 , no. 3 , pp. 677-689, 2012. 
[28] O. C. Imer, S. Yüksel, and T. Başar, "Optimal control of LTI systems over unreliable communication links," Automatica, vol. 42, no. 9, pp. 1429-1439, 2006.

[29] H. Xu, S. Jagannathan, and F. L. Lewis, "Stochastic optimal control of unknown linear networked control system in the presence of random delays and packet losses," Automatica, vol. 48, no. 6, pp. 1017-1030, 2012.

[30] E. Garone, B. Sinopoli, A. Goldsmith, and A. Casavola, "LQG control for MIMO systems over multiple erasure channels with perfect acknowledgment," IEEE Trans. Automat. Control, vol. 57, no. 2, pp. 450-456, 2012.

[31] Y. Mo, E. Garone, and B. Sinopoli, "LQG control with Markovian packet loss," in Control Conf. (ECC), 2013 European. IEEE, 2013, pp. 23802385.

[32] H. Lin, Z. Xu, H. Su, Y. Xu, and Z.-G. Wu, "Fast filtering algorithm for state estimation of lossy networks," IET Control Theory \& Applications, vol. 8, no. 18, pp. 2316-2324, 2014.

[33] B. Anderson and J. B. Moore, Optimal Filtering. Englewood Cliffs: Prentice-Hall, 1979.

[34] A. H. Jazwinski, Stochastic Processes and Filtering Theory. Academic Press, New York, 1970.

[35] H. Lin, H. Su, Z. Shu, Z.-G. Wu, and Y. Xu, "Optimal estimation for networked control systems with intermittent inputs without acknowledgement," in Proc. 19th IFAC World Congress, 2014, pp. 5017-5022.

[36] R. R. Bitmead and M. Gevers, "Riccati difference and differential equations: Convergence, monotonicity and stability," in The Riccati Equation. Springer, 1991, pp. 263-291.

[37] R. A. Horn and C. R. Johnson, Matrix Analysis. Cambridge university press, 2012.

[38] P. S. Maybeck, Stochastic Models, Estimation, and Control. Academic press, 1982, vol. 3. 